\title{
Integrated Dielectric Model for Unconsolidated Porous Media Containing Hydrate
}

\author{
Bin Wang, Member, IEEE, Zhonghao Zhang, Lanchang Xing, Member, IEEE, Liyun Lao, Zhoutuo Wei and Xinmin Ge
}

\begin{abstract}
This paper reports a novel dielectric model developed for estimating the complex permittivity of unconsolidated porous media containing gas hydrate. The complex permittivity spectra were experimentally obtained by using open-ended-coaxial-probes over a frequency range between $1 \mathrm{MHz}$ and $3 \mathrm{GHz}$, in which the dielectric dispersion of both hydrate and liquid solution are covered. With tetrahydrofuran used as the hydrate former, reflection coefficients were recorded during the hydrate formation and dissociation processes in quartz sands and the complex permittivity spectra were inversed. Volumetric fractions estimated from the $X$-ray-tomography were used as the referenced values. Experimental data showed that the Maxwell-Wagner effect, surface conductance, and phase configuration can affect the bulk permittivity. The discrepancy was found to be unacceptable when the fitting was conducted with pre-existing models such as ComplexRefractive-Index-Method and Maxwell-Wagner-BruggemanHanai. In this study, by modifying the nested Wagner's theory, a shell coated model was applied, and the surface of the solid sphere was assumed to possess surface conductance when it was humidified by the liquid solution. In contrast to Complex-Refractive-Index-Method and Maxwell-Wagner-Bruggeman-Hanai, the proposed model allows more accurate estimation of the volumetric fraction. By adopting this model with a range of dielectric measurements with different phase configurations, temperature, particle size, surface conductivity, and frequency, the contents of components and their influences onto the bulk permittivity can be physically estimated. The proposed model provides an essential tool for the interpretation of dielectric dispersion curves and the prediction of the volumetric fractions, which can be useful for both the field and laboratory applications.

Index Terms - Gas hydrate, Complex permittivity, Dielectric mixing law, Porous Media
\end{abstract}

This research was jointly supported by National Natural Science Foundation of China (51306212, 41704124), the Fundamental Research Funds for the Central Universities (16CX05021A, 18CX02112A and 18CX02176A), Shandong Provincial Natural Science Foundation (ZR2019MEE095, ZR2017BEE026) and Key project of PetroChina (ZD2019-184). Thanks to Dr. Edward C. Mignot, Shandong University, for linguistic advice.

Bin Wang is with College of Control Science and Engineering, China Unive rsity of Petroleum (East China), 266580, China (e-mail: wangbin2015@upc.ed u.cn).

Zhonghao Zhang is with College of Control Science and Engineering, Chin a University of Petroleum (East China), 266580, China (e-mail: zzzhhh_2008 @ 163.com).

\section{INTRODUCTION}

$\mathrm{G}^{\mathrm{a}}$ as hydrates are clathrate crystalline solid compounds consisting of gas molecules trapped in water cages[1]. The naturel gas hydrate could be a future unconventional gas resource, if its exploitation is environmentally friendly and technically recoverable [2]. Methane hydrate is abundant in many locations such as sea floor sediments which can be regarded as an unconsolidated porous medium and permafrost regions[3-5], and research on the identification and drilled gas hydrate has been conducted in many countries[6-9].

Geophysical well logging methods widely used in traditional oil and gas engineering were applied to gas hydrate exploration[7, 10-12]. The EPT (Electromagnetic Propagation Tool) working at $1.1 \mathrm{GHz}$ was reported to provide hydrate saturation in reservoirs in Alaska, and the inversion results had good consistency with other logging methods such as ultrasonic and electrical resistivity tools[13-15]. Compared to the electrical resistivity methods, the dielectric method has many advantages such as less prone to water salinity and better vertical resolution, which are desirable for hydrate reservoir evaluation of the sea floor sediments such as salt water invasion and quantitative evaluation of hydrate saturation in thin layers[16, 17]. Physical characteristics of unconsolidated porous media containing hydrate crystals are important to natural gas hydrate detection and exploitation from the sea bottom and tundra. Hence, many dielectric spectroscopic studies of the dielectric properties of porous media containing hydrates have been carried out in the laboratory [18-22], and moreover, studies of hydrates in oil and gas transportation have also been conducted[23]. Bulk permittivity both in the wide band and frequency points can be affected by hydrate fractions, and dielectric information was found to be a good indicator of hydrate formation and dissociation[24-26].

Calculating the dielectric properties of mixtures has been a challenging problem of both theoretical and practical importance. The bulk permittivity is always determined not

Lanchang Xing is with College of Control Science and Engineering, China University of Petroleum (East China), 266580, China (e-mail: xinglc@upc.ed u.cn).

Liyun Lao is with the School of Water, Energy, and Environment, Cranfield University, MK43 0AL, U.K. (email: 1.lao@cranfield.ac.uk).

Zhoutuo Wei is with School of Geosciences, China University of Petroleum (East China), 266580, China (e-mail: weizt@upc.edu.cn).

Xinmin Ge is with School of Geosciences, China University of Petroleum (East China), 266580, China (e-mail: gexinmin2002@upc.edu.cn). 
only by volumetric fractions of each component, but is also affected by other complicated factors, such as phase distribution, ion transportation, electric double layer, and interfacial polarization $[27,28]$. The situation is much more complicated in the frequencies below 100MHz. Many geophysicists extended the polarization behaviors to lower frequencies by relating to Maxwell-Wagner polarization effect[29]. Other approaches were based on the surface conductance mechanism with colloidal chemistry[30, 31]. Studies showed that the bulk permittivity of multi-phase mixtures can be predicted by dielectric mixing models applied in the microwave band [18, 21]. The dielectric spectrum of hydrate-bearing sand and sediments containing clay in $200 \mathrm{MHz}$ to $1.3 \mathrm{GHz}$ were obtained with the open-ended coaxial method, and by adopting the Complex-Refractive-Index-Method (CRIM) for the dielectric constant at $1 \mathrm{GHz}$. hydrate saturation during the phase transformation were calculated[18, 21]. The CRIM type models were also applied in field logging data inversion[13, 14]. The formation around the borehole was approximated as a homogenous and continuous medium, and the porous media were assumed to consist of multi components such as solid grains, hydrates, free water, and clay. When the CRIM was used as the fitting method, the dispersion properties at the frequency band below $100 \mathrm{MHz}$ were ignored[32]. However, gas hydrates have relaxation frequencies in the band from $0.1 \mathrm{MHz}$ to $100 \mathrm{MHz}$, which depends on guest molecules[33, 34]. Additionally, previous studies have proved that the presence of free charges and numerous pore filler such as liquid and clay grains, generate a Maxwell-Wagner effect that can critically affect the bulk dielectric measurement in the frequency band below $100 \mathrm{MHz}[35,36]$. Taking interfacial polarization into consideration, fitted calculations based on the MaxwellWagner-Bruggeman-Hanai(MWBH) method were used in predicting the dielectric spectrum during hydrate agglomeration and melting in emulsions, and the MWBH method has been extended to model the spherical particles with a confocal shell $[25,35,37]$. However, there has been no dielectric mixture model which is applicable in a broad frequency range from $\mathrm{MHz}$ to $\mathrm{GHz}$ for porous media containing gas hydrate yet reported, which is of interest in geophysics.

Previously we reported experimental work on the permittivity dispersion spectra of the unconsolidated porous media containing gas hydrate and the permittivity variation during the hydrate agglomerating and melting process, by using the open-ended coaxial method [38]. Because of the complete miscibility of tetrahydrofuran(THF) in water, stability under atmosphere pressure, analogous electrical properties with methane hydrate, and uniform spatial hydrate distribution, THF was widely used as the hydrate former instead of methane in laboratory studies[39].

In this paper we would report an extended study in which model based on Wagner's equation was developed to estimate the volumetric fractions of each component in unconsolidated quartz sands containing hydrate. To improve the performance of the model the solid surface conductance was also taken into consideration. Briefly the modelling procedures were as follows: First, the measured spectra were fitted with the proposed model during the process of hydrate formation and dissociation. Then the fitted performance of the proposed model at different experimental stages was compared with the CRIM and MWBH models. The volumetric fractions obtained with Xray $\mathrm{CT}$ were regarded as the standard value, and the prediction of the volumetric fraction obtained by the proposed model was more precise than the CRIM and MWBH models. Finally, variable factors such as temperature, surface conductivity, and particle size were taken into consideration, and model parameters were presented to aid the analysis of the interaction mechanism between the multiple components and the physical component transformation processes.

\section{THEORETICAL CONSIDERATIONS}

\section{A. Complex Refractive Index Method (CRIM)}

The CRIM model predicts the bulk permittivity by making a linear weighted sum of the complex permittivity square root of each component at a single frequency. In this study, the complex permittivities of the four components were together considered to influence the bulk permittivity $\varepsilon_{m i x}{ }^{*}$. As shown in (1), $\varepsilon_{\text {sand }}{ }^{*}, \varepsilon_{\text {solution }}{ }^{*}, \varepsilon_{\text {hydrate }}{ }^{*}$, and $\varepsilon_{\text {air }}{ }^{*}$ are complex permittivities of quartz sand, THF solution, THF hydrates, and air, respectively, and $A, B, C$, and $D$ represented volumetric fractions of the complex permittivity of quartz sand, THF solution, THF hydrates, and air, respectively.

$$
\sqrt{\varepsilon_{\text {mix }}^{*}}=A \sqrt{\varepsilon_{\text {sand }}^{*}}+B \sqrt{\varepsilon_{\text {solution }}^{*}}+C \sqrt{\varepsilon_{\text {hydrate }}^{*}}+D \sqrt{\varepsilon_{\text {air }}^{*}}
$$

The CRIM was initially proposed and validated in[40], and then was widely used in the interpretation of remote sensing and geophysical detection [41]. By taking the different performance of bounded water and free water, and to include the prediction of moist soil, the CRIM was extended as a generalized refractive mixing dielectric model (GRMDM)[32]. In recent years, the CRIM model has been applied in both laboratory hydrate-bearing sediments $[18,21]$ and reservoir evaluation of hydrate with the electromagnetic propagation tool (EPT)[13, 14]. This model became one of the most popular models in geophysics application, but it does not take interactions between the components in the lower frequency band into account, such as the Maxwell-Wagner effect (or M-W effect), electrochemical effects below $100 \mathrm{MHz}$, size and shape of the particles, and pore structure. For experiments in this study, liquid existed in several stages, and the structure and components of the mixture varied in the experimental process, which made the fitted performance of CRIM uncertain in a low frequency range.

\section{B. Approaches Considering the $M$ - $W$ and Electrochemical Effects}

A mixture composed of multiple components with different dielectric constants and electrical conductivities shows a dielectric dispersion due to the so-called interfacial polarization, which varied with texture, grain size, and solution salinity[42]. This phenomenon was first pointed out by Maxwell by means of a stratified model[43], but it did not possess practicality since the differences between the dispersed phase and continuous 
medium was left out in Maxwell's model[44]. Wagner explained that for a mixed system where spherical particles are sparsely distributed throughout a continuous medium, the complex permittivity of the heterogeneous mixture can be expressed by the following equation[29], by solving the Laplace equation with boundary conditions at the surface of the spheres:

$$
\varepsilon_{m i x}^{*}=\varepsilon_{m}^{*} \frac{2 \varepsilon_{m}^{*}+\varepsilon_{\mathrm{p}}^{*}-2 \phi\left(\varepsilon_{m}^{*}-\varepsilon_{\mathrm{p}}^{*}\right)}{2 \varepsilon_{m}^{*}+\varepsilon_{\mathrm{p}}^{*}+\phi\left(\varepsilon_{m}^{*}-\varepsilon_{\mathrm{p}}^{*}\right)}
$$

Where $\varepsilon_{m i x}{ }^{*}$ is the complex permittivity of the two-phase mixture. Dispersed particles $p$ and continuous medium $m$ correspond to permittivities $\varepsilon_{p}{ }^{*}$ and $\varepsilon_{m}{ }^{*}$, and the volumetric fractions $\Phi$ and 1- $\Phi$, respectively. If the conductivities at low frequency are taken into consideration, $\varepsilon_{p}{ }^{*}$ and $\varepsilon_{m}{ }^{*}$ are presented as:

$$
\begin{gathered}
\varepsilon_{m}^{*}=\varepsilon_{m}+j \frac{\sigma_{m}}{\omega \varepsilon_{0}} \\
\varepsilon_{p}^{*}=\varepsilon_{p}+j \frac{\sigma_{p}}{\omega \varepsilon_{0}}
\end{gathered}
$$

$\sigma_{p}$ and $\sigma_{m}$ are the conductivity of the dispersed phase and continuous phase, respectively. Many experimental studies have been carried out on the interfacial polarization. It has been certified that the same as other dispersions such as water molecular polarization, the interfacial polarization implied in the bulk permittivity can be mathematically described with the Cole-Cole formula:

$$
\varepsilon_{\text {mix }}^{*}=\varepsilon_{h}+\frac{\varepsilon_{l}-\varepsilon_{h}}{1+j \omega \tau}+\frac{q}{j \omega} \sigma_{\text {mix }}
$$

Where the relaxation time $\tau$, the high frequency dielectric constant $\varepsilon_{h}$, and its difference with low frequency dielectric constant of the mixture $\varepsilon_{l}-\varepsilon_{h}$ can be expressed as (making $\varepsilon_{m i x}{ }^{*}$ equal in (2) and (5)) [45]:

$$
\begin{gathered}
\tau=\frac{2 \varepsilon_{m}+\varepsilon_{p}+\phi\left(\varepsilon_{m}-\varepsilon_{p}\right) \varepsilon_{0}}{2 \sigma_{m}+\sigma_{p}+\phi\left(\sigma_{m}-\sigma_{p}\right)} \\
\varepsilon_{h}=\varepsilon_{m} \frac{2 \varepsilon_{m}+\varepsilon_{p}-2 \phi\left(\varepsilon_{m}-\varepsilon_{p}\right)}{2 \varepsilon_{m}+\varepsilon_{p}+\phi\left(\varepsilon_{m}-\varepsilon_{p}\right)} \\
\varepsilon_{l}-\varepsilon_{h}=\frac{9\left(\varepsilon_{m} \sigma_{p}-\sigma_{m} \varepsilon_{p}\right)^{2}}{\left[2 \varepsilon_{m}+\varepsilon_{p}+\phi\left(\varepsilon_{m}-\varepsilon_{p}\right)\right]} . \\
\frac{\phi(1-\phi)}{\left[2 \sigma_{m}+\sigma_{p}+\phi\left(\sigma_{m}-\sigma_{p}\right)\right]^{2}}
\end{gathered}
$$

It has been recognized from previous studies that Cole-Cole parameters $\tau$ and $\varepsilon_{h}$ are affected by the phase composition. However, when $\sigma_{p}$ is much less than $\sigma_{m}$ with high particle concentrations, the fitted bulk complex permittivity does not agree with the experimental data. Therefore, Wagner's theory is suitable only for a continuous media with dilute particle inclusions in which there is little interaction between particles.

For concentrated particle suspensions, it is difficult to rigorously solve the problem. An effective medium theory that provides a reasonable approximation to the problem was provided by Bruggeman[46]. In this model, the initially low content of the dispersed phase is gradually increased by an infinite-small addition of particles. After the small amount of particles with $\varepsilon_{p}$ are added, the mixture can be regard as a new homogeneous media with a refreshed bulk permittivity. At each step, the added amount of particles is infinitesimal, and then the mixture permittivity is obtained with (2), and the volumetric fractions are synchronously recalculated. By continuous additions of infinitesimal particles, the mixed system ultimately reaches the final volume ratio and permittivity. Then an integral equation is established, and by solving it, and the Bruggeman's model is obtained. The sophisticated derivation is skipped here, as it can be found in several classical references[46, 47]. The Bruggeman's model was extended to the condition of complex permittivity by Hanai[45], and the Maxwell-WagnerBruggeman-Hanai (MWBH) model was established. Especially for spherical particles, the MWBH model is presented as the following equation:

$$
1-\phi=\left(\frac{\varepsilon_{m i x}^{*}-\varepsilon_{p}^{*}}{\varepsilon_{m}^{*}-\varepsilon_{\mathrm{p}}^{*}}\right)\left(\frac{\varepsilon_{m}^{*}}{\varepsilon_{m i x}^{*}}\right)^{1 / 3}
$$

Both Wagner's model and the MWBH model can be extended to a three-phase system by simply setting up a confocal shell of the third phase between the spherical core and the external medium[48]. As shown in Fig. 1(a), the shelled spheres act as the dispersed phase possessing the inner cores with complex permittivity $\varepsilon_{1}{ }^{*}$ and the outer shells with $\varepsilon_{2}{ }^{*}$, and the effective complex permittivity of the spheres $\varepsilon_{p}{ }^{*}$ can be presented as:

$$
\varepsilon_{p}^{*}=\varepsilon_{2}^{*} \frac{2 \varepsilon_{2}^{*}+\varepsilon_{1}^{*}-2 \phi^{\prime}\left(\varepsilon_{2}^{*}-\varepsilon_{1}^{*}\right)}{2 \varepsilon_{2}^{*}+\varepsilon_{1}^{*}+\phi^{\prime}\left(\varepsilon_{2}^{*}-\varepsilon_{1}^{*}\right)}
$$

Where the $\Phi$ ' is the volumetric fraction of the inner cores of the shelled spheres:

$$
\phi^{\prime}=\left(\frac{4 \pi}{3} r^{3}\right) /\left(\frac{4 \pi}{3} R^{3}\right)=\left(\frac{r}{R}\right)^{3}
$$

By substituting (10) into (9), the final effective mixture permittivity $\varepsilon_{\text {mix }}{ }^{*}$ for a three-phase system is obtained. Similarly, a combination of (10) and (2) can also provide a prediction of effective permittivity for a dilute dispersion system. In addition, the three-phase models can be further extended to more complicated multi-components systems[49-51].

In this study, in order to fit the complex permittivity of unconsolidated porous media containing hydrate and to estimate volumetric fractions, we defined a confocal multi-shell sphere model as shown in Fig. 1(b) to illustrate the procedure. The sphere includes $n$ layers of confocal shells numbered from 1 to $n$ in the radial direction between the inner core and the continuous phase. For the core covered with the first shell, the equivalent complex permittivity of the particle $\varepsilon_{s 1}{ }^{*}$ is given by:

$$
\varepsilon_{p 1}^{*}=\varepsilon_{s 1}^{*} \frac{2 \varepsilon_{s 1}^{*}+\varepsilon_{c}^{*}-2 \phi_{1}^{\prime}\left(\varepsilon_{s 1}^{*}-\varepsilon_{c}^{*}\right)}{2 \varepsilon_{s 1}^{*}+\varepsilon_{c}^{*}+\phi_{1}^{\prime}\left(\varepsilon_{s 1}^{*}-\varepsilon_{c}^{*}\right)}
$$

Where $\varepsilon_{c}{ }^{*}$ and $\varepsilon_{p l}{ }^{*}$ are the complex permittivity of the core and the first shell, respectively and $\Phi^{\prime}{ }_{1}$ is the inner core's volumetric fraction. Then, the particle with the $1 s t$ layer of the shell is regarded as a new homogeneous inner phase with volumetric fraction $\Phi_{2}{ }_{2}$, and the effective complex permittivity of the particle with the $2 n d$ shell is represented as: 


$$
\varepsilon_{p 2}^{*}=\varepsilon_{s 2}^{*} \frac{2 \varepsilon_{s 2}^{*}+\varepsilon_{p 1}^{*}-2 \phi_{2}^{\prime}\left(\varepsilon_{s 2}^{*}-\varepsilon_{p 1}^{*}\right)}{2 \varepsilon_{s 2}^{*}+\varepsilon_{p 1}^{*}+\phi_{2}^{\prime}\left(\varepsilon_{s 2}^{*}-\varepsilon_{p 1}^{*}\right)}
$$

Circularly, with a similar analogy, for the spherical particle with $n$ layers of shells, its equivalent complex permittivity $\varepsilon_{p n}{ }^{*}$ can be obtained with $\varepsilon_{p(n-1)}{ }^{*}, \varepsilon_{s n}{ }^{*}$, and $\Phi_{n}^{\prime}$. Finally, substituting $\varepsilon_{p n}{ }^{*}$ into (2), the bulk permittivity can be calculated with this nested Wagner model (the confocal multi-shell sphere model is called the NW model, for short).

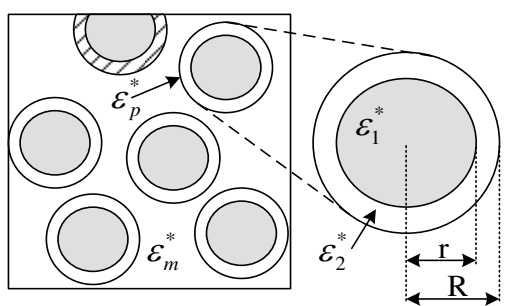

(a)

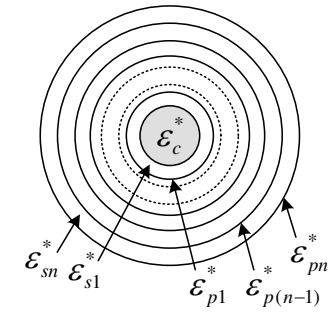

(b)
Fig. 1. Electrical models for three-phase systems and multi-shell systems, and the schematic diagrams derivation procedure of the theoretical equations. (a) the three-phase system where $r$ represents the inner core's radius and $R$ represents the outer radius of the shell, (b) the multi-shell model.

\section{Modification with Solid Surface Conductance}

The M-W effect is not the exclusive contribution to the lowfrequency dielectric dispersion of the grain packs containing liquid. The other contribution is the electrical double layer coating the surface of grains: the electrochemical effect[31]. A fixed charge density is located at the surface of grains in contact with the water solution, and the conductance in electrical double layers is called the surface conductance. The surface conductance $\sum_{s}$ can be written as the sum of two parts[52]:

$$
\sum_{S}=\sum_{S}^{E D L}+\sum_{S}^{S t e r n}
$$

Where $\sum_{S}^{E D L}$ and $\sum_{s}^{\text {Ster }}$ are the contributions from the ionic conduction in the electrical diffuse layer (outer layer) and in the Stern layer (inner part of the double layer), respectively. For very low salinity, the first part $\sum_{s}^{E D L}$ is a minor component compared to $\sum_{s}^{\text {stem }}$, which is formed by ions adsorbed on the solid surface. The double layer effect is believed to play the dominate role in a frequency range lower than the $\mathrm{M}-\mathrm{W}$ effect. However, in the intermediate frequency range $(10 \mathrm{kHz}$ to $10 \mathrm{MHz}$ ), where both the M-W and electrochemical effects exist, many theoretical approaches have been proposed to interpret the experimental data, e.g. extending the M-W mechanism to lower frequencies and establishing standard models of colloidal chemistry[53]. In the geophysical literature, the low frequency range is envisioned to be dominated by the $\mathrm{M}-\mathrm{W}$ effect, which is due to the discontinuity of the displacement current at the interface between different components. Counterions in the Stern layer can move tangentially to the mineral surface, and beyond that they attain some degree of freedom to get sorbed and desorbed[54]. M-W polarization and surface conductance should be considered together in the intermediate frequency range [55-59], and they are both affected by the porous media's geophysical properties, e.g. grain size distribution and the mineral surface[57, 60]. As a side note, the neglect of the surface conductance leads to spurious interpretations, and in quartz sands, it cannot be ignored at very low salinities[61].
The CRIM, Wagner, or the MWBH models do not consider the surface conductance with an assumption that the conductivity of the solid phase is negligibly small. In this study, we took the surface conductance contributed by the electrical double layers into consideration, and the NW model was modified by introducing the surface conductivity to the outermost layer of the solid phase.

\section{EXPERIMENTAL METHOLOGY}

\section{A. Complex Permittivity}

The complex permittivity is a combination of the material's dielectric constant and loss factor, and both its real and imaginary spectrum are always changing with frequency. Taking the conductivity into account, the complex permittivity can be expressed as:

$$
\varepsilon^{*}=\varepsilon^{\prime}-j \varepsilon^{\prime \prime}-j \frac{\sigma}{\omega \varepsilon_{0}}
$$

Where $\varepsilon^{*}$ is the complex relative permittivity, and $\varepsilon$ and $\varepsilon$ " are the dielectric constant and dielectric loss, respectively, and $\sigma, \varepsilon_{0}, \omega$, and $j$ are conductivity, vacuum dielectric constant, angular frequency and the imaginary unit, respectively. In this study, the mixture is composed of the liquid THF solution, THF hydrate, quartz sand, and air together or partially depending on the experimental process. Thus, to illustrate the differences between dielectric properties of the four components, the dielectric constant and loss factor spectra at a frequency range from $1 \mathrm{kHz}$ to $10 \mathrm{GHz}$ are schematically presented in Fig. 2 . Both THF solution and THF hydrate have a prominent dispersion, compared with the invariable spectra of air and quartz sand. The dielectric constant is related to the net polarization opposing the applied EM field, and the loss factor $\left(\varepsilon "+\sigma / \omega \varepsilon_{0}\right)$ is interpreted as the joule heat loss and polarization loss. Different polarization phenomena such as relaxation of polar molecules, the Maxwell-Wagner effect, and the displacement of atoms' electronic cloud result in the dispersion of the complex permittivity [62]. In clathrate hydrates the dispersion peak which is located below $10 \mathrm{MHz}$, theoretically depends on the properties of the encaged guest molecules, and it always overlaps the interfacial polarization in the frequency domain[34]. The permittivity spectrum of THF solution is affected by the solution proportion, and a predominate dispersion caused by molecular polarization can be observed over $1 \mathrm{GHz}$. Moreover, it was reported that both the spectra amplitude and dispersive frequency of the hydrate and solution vary with temperature [63-65]. In the displayed frequency range, the dielectric constant of quartz sand and air are stable, and their loss factors are both negligibly small. In brief, the existence of THF solution and THF hydrate can be determined with their specific dispersion of complex permittivity. 


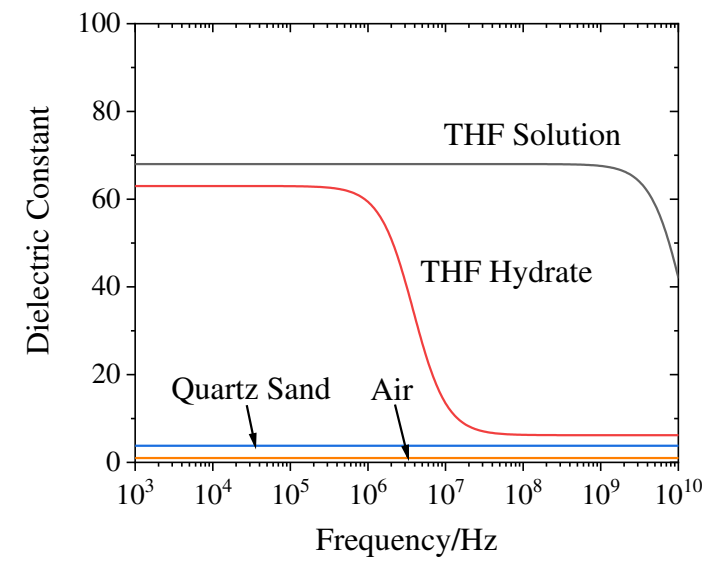

(a)

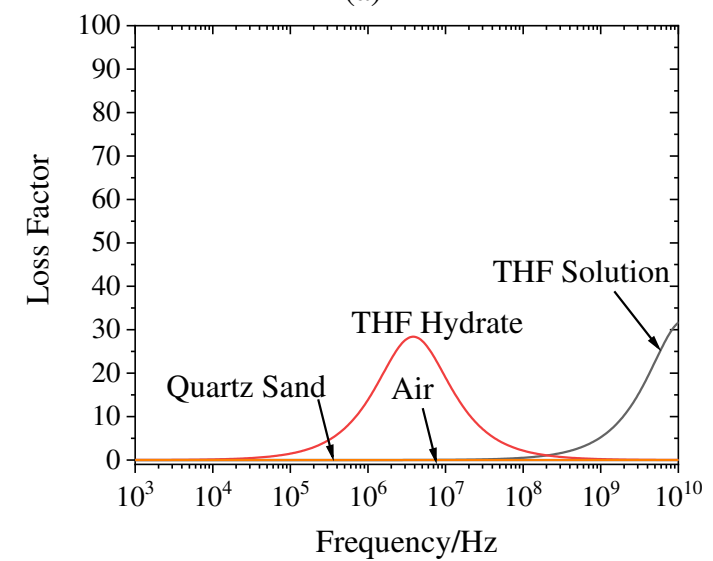

(b)

Fig. 2. Dielectric constant (a) and loss factor (b) of the complex permittivity of quartz sand (blue), the THF hydrate (red), air (orange), and THF solution (black).

\section{B. Dielectric Measurement}

The complex permittivities in this study were obtained with a dielectric measurement system which is the same as the equipment used in our previous work[38]. The dielectric probe acts as the key-part of the system and its detailed structure is shown in Fig. 3. The length of in-house made open-ended coaxial dielectric probe is $200 \mathrm{~mm}$, and its open section's transverse dimension are presented in the subgraph of Fig. 3 (outer diameter of inner conductor $2 \mathrm{a}=0.51 \mathrm{~mm}$, the inner diameter of outer conductor $2 b=1.67 \mathrm{~mm}$, and these two parameters will be used in complex permittivity inversion). A Pt100 resistance temperature detector (RTD) probe was bound with the slim type dielectric probe, and the RTD's terminal was a little higher than the open end of the dielectric probe, so that the interference of the metal resistance element to dielectric measurement could be avoided. The integrated probes penetrated into the measured medium, the dielectric probe and RTD were connected with the vector network analyzer (VNA type 5061B from Keysight) and temperature recorder (type IV3000 from IVYTECH). In the whole experimental process, the vector network analyzer and the temperature recorder were controlled by a master computer to periodically record both the reflection coefficient from $1 \mathrm{MHz}$ to $3 \mathrm{GHz}$ and temperature. The dielectric spectra were inversed from the complex reflection coefficient, and this calculation will be discussed in the next section.

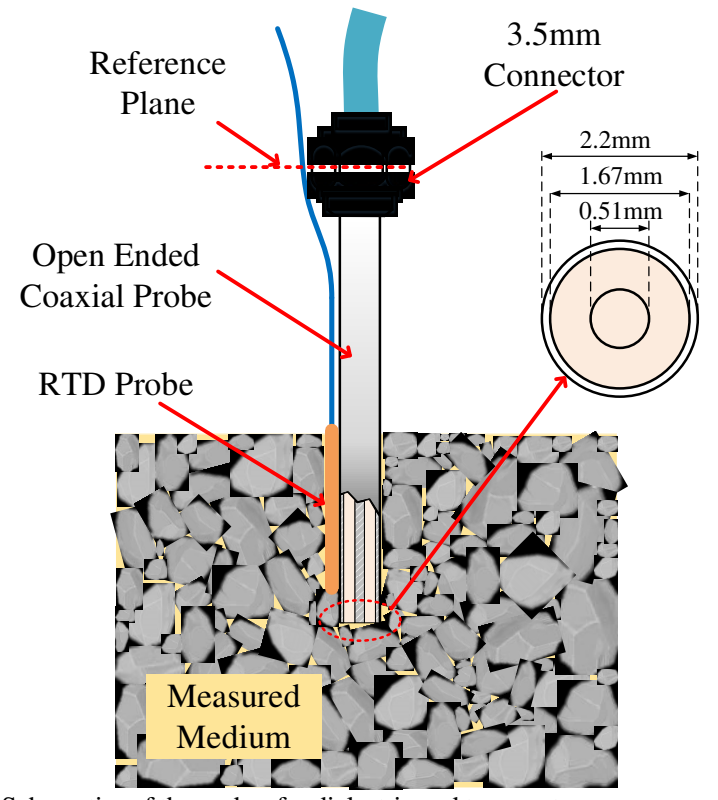

Fig. 3. Schematics of the probes for dielectric and temperature measurement. The open-ended coaxial dielectric probe and the RTD probe are bound together and penetrated into the measured medium. The detailed structure of the open-ended probe is shown in the subgraph at the right side.

The THF (>99.9\%) and deionized water were mixed as the hydrate former, and the mass ratio was $1: 4.25$ that guarantees no liquid remained after the hydrate formed. Quartz sand with two particle sizes (to simplify the calculation, quartz sand particles with diameter about $0.9 \mathrm{~mm}$ and about $0.28 \mathrm{~mm}$ were homogenized as spheres with diameters of $0.9 \mathrm{~mm}$ and $0.28 \mathrm{~mm}$ ) was prepared as the porous media, and the sand was carefully washed to make the residual soluble ions as few as possible. This is because the pore liquid salinity not only affects the permittivity of the mixture, also can change the formation process and equilibrium condition of the hydrate[66-68]. In this study, salinity was kept extremely low to diminish its influence, and the effect of the pore liquid salinity will be studied in our following works. The THF solution was added into the quartz sand until supersaturated. The electrical conductivity of the pore liquid was monitored with the TDS probe and conductance meter, and the conductivity was less than $1 \mathrm{e}-5 \mathrm{~S} / \mathrm{m}$ which was at least one order smaller than surface conductivity in this study. All the mixtures were placed together into a glass reactor which was sealed with a rubber plug and placed inside the computer-controlled thermotank.

\section{Inversion of the Complex Permittivity}

Before the measurement of complex permittivity was carried out, the VNA was calibrated with calibration kits (type $85033 \mathrm{E}$ from Keysight), and as shown in Fig. 3, its reference plane was located at the $3.5 \mathrm{~mm}$ connector which links the dielectric probe and the phase stable cable. The electromagnetic wave with transverse electric and magnetic (TEM) mode incident on the open end of the dielectric probe was partially reflected back, with a part of it radiated into the material attached to the terminal. Simultaneously, the incident wave generated many other higher order modes over the annular open end[69]. The complex reflection coefficient is significantly affected by the dielectric and loss properties of the material under test (MUT). 
When the size of the probe terminal aperture is electrically small, the relationship between complex reflection coefficient $\Gamma^{*}$ and the complex permittivity of MUT $\varepsilon_{s}{ }^{*}$ can be established with the quasi-static model in the frequency domain[70]:

$$
\left\{\begin{array}{l}
\frac{1-\Gamma^{*}}{1+\Gamma^{*}}=\frac{\sqrt{\left(\mu_{0} \mu_{1}\right) /\left(\varepsilon_{0} \varepsilon_{1}\right)}}{\ln (b / a)} c \\
c=\int_{0}^{\infty} \frac{\left[J_{0}\left(k_{c} a\right)-J_{0}\left(k_{c} b\right)\right]^{2}}{\left[1-2 \exp \left(-\sqrt{k_{c}^{2}-\omega^{2} \varepsilon_{0} \varepsilon_{s}^{*} \mu_{0} \mu_{s}} d\right]\right.} . \\
\frac{\left[1+2 \exp \left(-\sqrt{k_{c}^{2}-\omega^{2} \varepsilon_{0} \varepsilon_{s}^{*} \mu_{0} \mu_{s}} d\right] \varepsilon_{s}^{*} i \omega \varepsilon_{0}\right.}{k_{c} \sqrt{k_{c}^{2}-\omega^{2} \varepsilon_{0} \varepsilon_{s}^{*} \mu_{0} \mu_{s}}} d k_{c}
\end{array}\right.
$$

Where $a$ is the outer radius of the inner conductor (mm), $b$ is the inner radius of the outer conductor $(\mathrm{mm}), d$ is the effective thickness of the MUT, $k_{\mathrm{c}}$ is the continuous eigenvalue which acts as the integrating factor, $\varepsilon_{0}$ and $\mu_{0}$ are the permittivity and magnetic permeability of vacuum, respectively, $\varepsilon_{1}$ and $\mu_{1}$ are the relative permittivity and relative magnetic permeability of the dielectric probe's filling material, respectively, (the cylindrical dielectric material of our probe was Teflon with $\mu_{1}$ $=\mu_{\mathrm{s}}=1$ and $\varepsilon_{1}=2.1$ ), and $\varepsilon_{\mathrm{s}}$ and $\mu_{\mathrm{s}}$ are the relative complex permittivity and relative magnetic permeability of the MUT, respectively.

Although the characteristic impedance of the open-ended probe was $50 \mathrm{Ohm}$, insertion loss of the $3.5 \mathrm{~mm}$ connector and the probe's length can both introduce errors into the complex reflection coefficient $\Gamma^{*}$. The systematic error of directivity $\left(e_{\mathrm{d}}\right)$, frequency response error $\left(e_{\mathrm{r}}\right)$, and equivalent source match error $\left(e_{\mathrm{s}}\right)$ gave rise to deviations between the reflection coefficient measurement by the VNA $\Gamma_{\mathrm{m}}$ and the actual reflection coefficient $\Gamma_{\mathrm{a}}$. Compensation corrections by measuring three materials with known reflection coefficients provided a solution for the systematic errors in the frequency domain. Then the three systematic errors were mathematically removed from subsequent permittivity measurements. $\Gamma_{\mathrm{a}}$ is derived from the following equation, and it equals the complex reflection coefficient $\Gamma^{*}$ in (15)[71]:

$$
\Gamma_{\mathrm{a}}=\frac{\Gamma_{\mathrm{m}}-e_{\mathrm{d}}}{e_{\mathrm{s}}\left(\Gamma_{\mathrm{m}}-e_{\mathrm{d}}\right)+e_{\mathrm{r}}}
$$

In this study, the compensation was carried out by using $25^{\circ} \mathrm{C}$ deionized water, a short circuit kit with gold plated rubber, and air. The permittivity of pure water at standard atmospheric pressure and the temperature of $25^{\circ} \mathrm{C}$ was adopted as the referenced permittivity of the $25^{\circ} \mathrm{C}$ deionized water [72], and the reflection coefficients with air and deionized water were derived with (15). The inversion method for open-ended coaxial probe was established, and the complex permittivities in a very wide frequency range $(1 \mathrm{MHz}-3 \mathrm{GHz})$ were obtained with the open-end coaxial method.

\section{Validation of Volumetric Fraction Results}

In order to further verify the accuracy of the fitted results, we build the standard of volumetric fractions with an X-ray CT system ('SOMATOM Definition AS' from SIEMENS with $100 \mu \mathrm{m}$ resolution). The glass reactor containing the mixed media was scanned before the hydrate's formation started (state without the hydrate-WHE) and after its formation was completed (state of no liquid solution left-NLL). In the following studies, the standard values of volumetric fractions were quantitatively obtained with this system in the two experimental states. The scanning time for each sample was about $1 \mathrm{~min}$, which was adequately short to prevent the hydrate from unwanted melting. The distribution of the mixture was approximatively homogeneous, and a short cylindrical area (radius $\mathrm{r}=1 \mathrm{~cm}$, height $\mathrm{h}=0.5 \mathrm{~cm}$ ) was selected around the probes' terminal and false-colored with Matlab. The reconstructed 3D images are presented in Fig. 4, where the top horizontal plane is parallel to the open end of the coaxial probes which penetrated about $1 \mathrm{~cm}$ into the mixture, and the particle size was about $0.9 \mathrm{~mm}$. The reversed results slightly varied with the size of the sand particles. For the sake of brevity, the X-ray CT image with a particle size of about $0.28 \mathrm{~mm}$ are not shown. The standard value of volumetric fractions can be found in Table I. The green color indicates the quartz sand particles, the blue and yellow inclusions indicate the fluid solution and THF hydrate, respectively, and the black color is the air which can be observed more clearly in the sub-figure of Fig 7(b). The dimension of the thermotank's outline exceeded the scanning area, and what is more, the electric circuit of the X-ray CT system cannot function below $4.4^{\circ} \mathrm{C}$. Due to these limitations, the volumetric fraction standard values of experimental stages during the formation and dissociation processes of the hydrate which occurred below $4.4^{\circ} \mathrm{C}$ can only be speculated from data at WHE and NLL states.

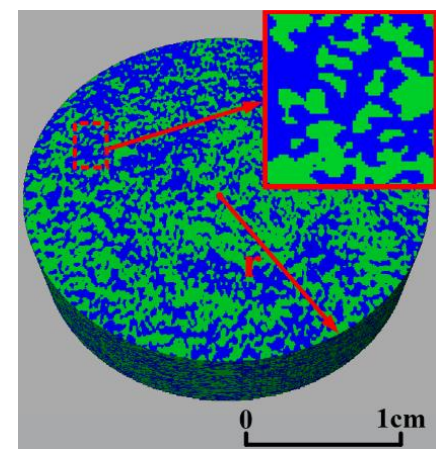

(a)

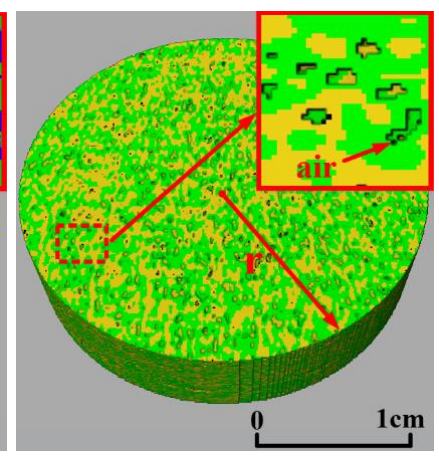

(b)
Fig. 4. The reconstructed 3D X-ray CT images with Matlab. Blue, green, black, and yellow represent THF solution, quartz sand, air, and THF hydrate respectively. The radius of the selected cylinder $r=1 \mathrm{~cm}$, and the particle size is about $0.9 \mathrm{~mm}$. (a) Image before THF hydrate's formation started, (b) image after THF hydrate's formation was completed.

From the X-ray CT analysis, it can be seen that the distribution of the mixtures is basically uniform in the vicinity of the probe's open end. As presented in Table I, the inversed volumetric fraction of samples with different particle sizes are roughly the same. For each particle size, the gas phase inclusion appeared after the hydrate formation had been completed (as shown in Fig. 4(b)), even though the gas phase content was critically small (see Table I) which came from the air initially dissolved in the THF solution[73, 74]. Furthermore, there was a slight decrease in the fraction of quartz sand in the NLL state. This is because that with the agglomeration of the hydrate the boundary of the hydrate cluster gradually expanded, and the bulk volume of the mixture increased. At the same time, with the liquid consumed, a tiny amount of air inclusions dissolved 
in the solution initially appeared. It can be observed from the subgraph of Fig. 4(b) that many air inclusions existed as a membrane covering the hydrate and sand particles. The proposed mixed model was applied to the quartz sands contacting the hydrate, and compared to the X-ray CT results, the fitted or prediction performance will also be estimated based on the standard values in Table I.

TABLE I

THE STANDARD VALUES OF VOLUMETRIC FRACTIONS

\begin{tabular}{cccccc}
\hline \hline \multirow{2}{*}{$\begin{array}{c}\text { Particle } \\
\text { Size(mm) }\end{array}$} & State & \multicolumn{4}{c}{ Volumetric Fractions } \\
\cline { 3 - 6 } & & $\begin{array}{c}\text { Quartz } \\
\text { Sand }\end{array}$ & $\begin{array}{c}\text { THF } \\
\text { Solution }\end{array}$ & $\begin{array}{c}\text { THF } \\
\text { Hydrate }\end{array}$ & \multirow{2}{*}{ Air } \\
\hline 0.90 & WHE & $45 \%$ & $55 \%$ & $0 \%$ & $0 \%$ \\
0.28 & WHE & $47 \%$ & $53 \%$ & $0 \%$ & $0 \%$ \\
0.90 & NLL & $44 \%$ & $0 \%$ & $53 \%$ & $3 \%$ \\
0.28 & NLL & $45 \%$ & $0 \%$ & $54 \%$ & $1 \%$ \\
\hline
\end{tabular}

\section{RESULTS AND ANALYSIS}

This section is divided into two parts. First, experimentation with the complete process of hydrate formation and dissociation will be presented. The nested Wagner's theory was modified with the surface conductance theory, and then it was applied to fit the time-varying measured dielectric spectrum at some representative stages. The fitted performance will be compared with the CRIM and MWBH model which has been commonly used in bulk dielectric prediction of porous media. In the second part, the effect of several factors, temperature, surface conductivity and particle size on the proposed model will be examined. The experimentation results showed that, the proposed NW model has demonstrated an excellent performance. It is capable of providing a good estimation of the components' fraction in the mixtures, under a number of changing influencing conditions.

\section{A. Fitting Performance of Different Phase Configuration}

Saturation is associated with a complex configuration of liquid, solids and gas phases in pore space in the unconsolidated quartz sand, which play an important role in complex permittivity evaluation. In this part, the measured complex permittivity of a representative experiment will be presented, and the spectra recorded at each experimental stage with different component configurations will be discussed. In the typical experiment, the quartz sand with a particle size of about $0.9 \mathrm{~mm}$ was used as the unconsolidated porous media, and the THF solution was added to supersaturate it. For the phase transition of gas hydrate, temperature was both the control means and status indicator, especially for THF hydrate which is stable at atmosphere pressure (phase equilibrium temperature of THF hydrate is $\left.4.4^{\circ} \mathrm{C}[18,20]\right)$. Fig. 5 shows the measured dielectric constant and loss factor at three typical frequencies $(3.5 \mathrm{MHz}, 300 \mathrm{MHz}$, and $3000 \mathrm{MHz})$ and the recorded temperature as a function of time during the whole experimental process.

The whole experimental process was divided into six stages, and significantly different characteristics were found with permittivity and temperature at each stage:

For stage A, the temperature was controlled dropping from room temperature until reaching around $0^{\circ} \mathrm{C}$, which was obviously a supercooled temperature for THF hydrate. During this stage, the mixture was in the 'WHE' state, and it can be seen from Fig. 5 that the complex permittivity changed with temperature. This is due to the temperature sensitivity of the liquid solution's dielectric properties, and moreover, analogous details were discussed in our previous publication [38], which will not be repeated here.

Stage A was terminated with drastic changes in both temperature and complex permittivity, which indicate the quick formation of hydrate around the probe, also there was liquid solution leaving the area far from the probe. Then the temperate recorded by the RTD was kept at $4.4^{\circ} \mathrm{C}$ for about 100 mins in stage $\mathrm{B}$, even though the temperature inside the thermotank continued dropping. Another interesting fact is that the dielectric constant at $3.5 \mathrm{MHz}$ kept pace with the RTD temperature, and a reverse process was found in stage $\mathrm{D}$. This is because of the temperature dependence of the hydrate dielectric dispersion at low frequency, which has already been previously clarified[38]. As the hydrate formation was completed in the whole reactor, the RTD temperature remained stable around $-10^{\circ} \mathrm{C}$ which is the lower limit of the target temperature. As a consequence, the mixture was in the 'NLL' state during stage $\mathrm{C}$.

Compared to the quick formation, the time of the dissociation process was much longer. During stage $\mathrm{E}$, the temperature was kept at $4.4^{\circ} \mathrm{C}$, the equilibrium temperature of THF hydrate, but which was different from stage $\mathrm{B}$ in that the complex permittivity at all three frequencies varied with time and showed an explicit dispersion. According to our experiments, temperature did not break the equilibrium $4.4^{\circ} \mathrm{C}$, until the hydrate in the whole reactor melted. However, it can be seen from the dielectric change that for the area inside the detection zone of the dielectric probe (about several millimeters adjacent to the probe's terminal), the dissociation process was concentrated in the time period between $746^{\text {th }}$ to $758^{\text {th }}$ min which is colored with dark grey in Fig. 5. When the equilibrium temperature was broken, it was converted to stage F, which returned to the 'WHE' state.

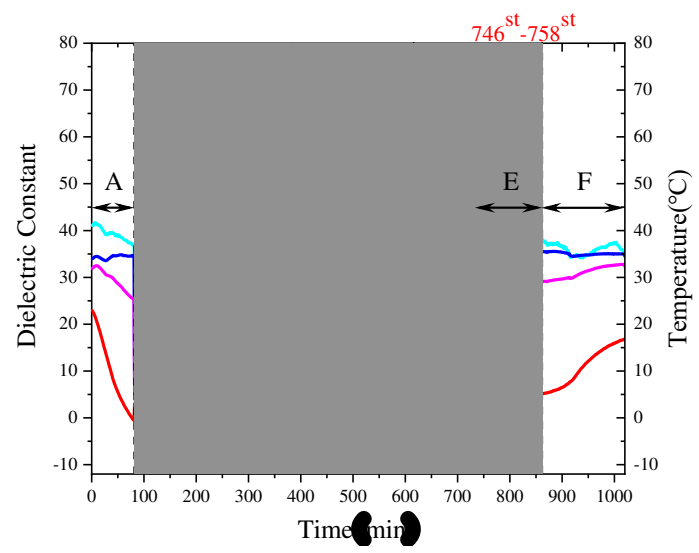

(a) 


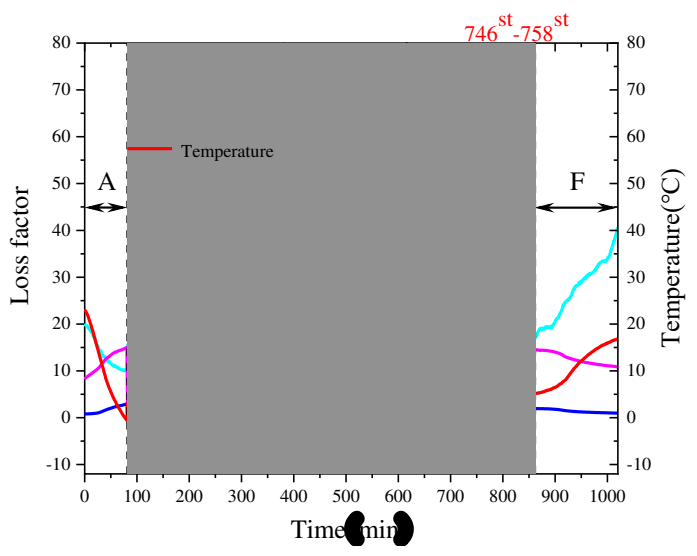

(b)

Fig. 5. Measured dielectric constant and loss factor at $3.5 \mathrm{MHz}, 300 \mathrm{MHz}$, and $3 \mathrm{GHz}$, and the recorded temperature during a representative experiment. Quartz sand was selected with a particle size of about $0.9 \mathrm{~mm}$. Corresponding to distinct states of the mixture, the whole experimental process was divided into six parts with dashed vertical lines. The light gray area indicates the existence of hydrate in the whole reactor, and the hydrate dissociation process occurred inside the detection area of the dielectric probe, concentrated in the dark gray area.

Estimation of component contents based on permittivity data can be reliable when a suitable mixing model is applied. CRIM, MWBH, and the modified NW model were used to fit the measured complex permittivity curve at stage A, B, D, E, and F. The first two models were used in previous studies on the hydrate fraction calculation, and the modified NW model proposed in this study was first adapted to hydrate estimation. $\sigma_{P S}$ represents the surface conductivity of the solid phase, and the conductivity of liquid solution was set as zero, because the THF solution is prepared with deionized water which can provide few free ions, and what is more, this was verified by applying the conductivity meter in stage $\mathrm{A}$ and $\mathrm{F}$.

In the microwave range, the non-dispersive relative dielectric constant of pure quartz sand $\left(\mathrm{SiO}_{2}>99.9 \%\right)$ was $\varepsilon_{\text {snad }}=4$ and it had a negligibly small loss factor[75]. The complex relative permittivity of THF solution $\varepsilon_{\text {solution }}^{*}$ and THF hydrate $\varepsilon^{*}$ hydrate can both be calculated with the Cole-Cole model:

$$
\varepsilon^{*}(\omega)=\varepsilon^{\prime}-j \varepsilon^{\prime \prime}=\varepsilon_{\infty}+\frac{\varepsilon_{s}-\varepsilon_{\infty}}{1+(j \omega \tau)^{1-\alpha}}-j \frac{\sigma}{\omega \varepsilon_{0}}
$$

Where $\varepsilon^{*}(\omega)$ is the dispersive complex permittivity of either THF solution or THF hydrate, $\varepsilon^{\prime}, \varepsilon^{\prime \prime}, \varepsilon_{s}$, and $\varepsilon_{\infty}$ are the dielectric constant, loss factor, dielectric constant at static, and infinity frequency, respectively, $\omega$ is the angular frequency, $\alpha$ is an index parameter which is less than $1, \tau$ is the relaxation time, and $\sigma$ is the conductivity. According to former study results, complex permittivity of both the liquid solution and hydrate showed obvious dependence on temperature [63-65]. As mention above, the conductivity of the liquid solution was set as zero. Moreover, the dielectric dispersion of THF solution is analogous to liquid water, and it was crucially influenced by the proportion of the solution. The relationship between parameters $\alpha, \tau, \varepsilon_{s}, \varepsilon_{\infty}$, and the temperature were illustrated in our previous study [38]. Specifically, the parameters of the Cole-Cole model that were used in this part are presented in Table II.

TABLE II

COLE-COLE PARAMETERS OF THF HYDRATE AND SOLUTION

\begin{tabular}{|c|c|c|c|c|c|c|c|c|c|}
\hline \multirow{2}{*}{ Stage } & \multirow{2}{*}{ Temperature } & \multicolumn{4}{|c|}{ THF Hydrate } & \multicolumn{4}{|c|}{ THF Solution } \\
\hline & & $\varepsilon_{\infty}$ & $\varepsilon_{s}$ & $\alpha$ & $\tau(s)$ & $\varepsilon_{\infty}$ & $\varepsilon_{s}$ & $\alpha$ & $\tau(s)$ \\
\hline A & $6.6^{\circ} \mathrm{C}$ & None & None & None & None & 5.85 & 67.5 & 0 & $1.59 \mathrm{e}-11$ \\
\hline B & $-1.1^{\circ} \mathrm{C}$ & 7 & 61.77 & 0.018 & $5.65 \mathrm{e}-8$ & None & None & None & None \\
\hline D & $-10^{\circ} \mathrm{C}$ & 7 & 63.62 & 0.018 & $8.12 \mathrm{e}-8$ & None & None & None & None \\
\hline E & $4.4^{\circ} \mathrm{C}$ & 7 & 60.7 & 0.018 & $4.59 \mathrm{e}-8$ & 5.85 & 67.8 & 0 & $1.65 \mathrm{e}-11$ \\
\hline $\mathrm{F}$ & $6.6^{\circ} \mathrm{C}$ & None & None & None & None & 5.85 & 67.5 & 0 & $1.59 \mathrm{e}-11$ \\
\hline
\end{tabular}

The fitted results and the measured data are shown in Fig. 6 and Fig. 7, and the estimated volumetric fractions and other parameters are listed in Table III. The dielectric properties at stage $\mathrm{C}$ were quite similar to stage $\mathrm{B}$, because in the region inside the detection range of the open-end probe, the mixtures at the two stages were both in the 'NLL' state. So it is not necessary to present the fitted results at stage $C$. The measured dielectric constant and loss factor are depicted as little dots in Fig. 6 and Fig. 7 in the whole experimental frequency range. For the band below $3 \mathrm{MHz}$, obvious distortion could be found, which is attributed to the performance limitation of the openended coaxial method. To evaluate the uncertainty of the measured results, the absolute value of the error calculated with the moving average are presented as bar graphs in Fig. 6 and Fig. 7. The amplitude of the uncertainties significantly rose below $3 \mathrm{MHz}$, therefore, only data measured over $3 \mathrm{MHz}$ were taken into consideration in this study. For stage A and F (both in the WHE state), two permittivity dispersions can be observed before the hydrate formation begins or after the dissociation is complete. One of them is the interfacial polarization caused by the M-W effect below $100 \mathrm{MHz}$, and the other is molecular polarization due to the dipole relaxation of the liquid solution over $1 \mathrm{GHz}$. For frequencies higher than $100 \mathrm{MHz}$, the fitted performances were all acceptable with the three models, which can also be extended to other stages. However, regarding the frequency lower than $100 \mathrm{MHz}$, the CRIM which is a mixture law based on the weighted sum of the complex refractive index, does not take the M-W effect caused by the migration of ions at low frequency into consideration. For the 'WHE' state, we consider a two-phase configuration where quartz sand particles are embedded in a liquid solution background. Then the MWBH and the NW model were simplified to (9) and (2) respectively, and the dispersed phase is the quartz sand. Equation (9) which is not a simple linear summation is complicated by the $1 / 3$ power and integrations. Even if all the particle sizes of the dispersed phase are set to a unified profile, it is still not easy to be solved with the algebraic method. Moreover, the THF solution that acts as the continuous background was blended with pure THF and deionized water, and it was almost an electrical insulation. Consequently, the 
fitted results with CRIM and MWBH kept consistent as the frequency decreased in stage A and F. To match the dispersion near the lower limit of the frequency band, conductance at the solid surface was employed in this study. In stage A, E, and F, when the surface of the solid phase was humidified by the solution, there was surface conductivity assigned to the particles with an order of $\mathrm{mS} / \mathrm{m}$ in the modified NW model which was believed caused by the electric double layer effect. As a result, in terms of dielectric dispersion at lower frequency (below 100MHz), the modified NW model achieved the best fitted performance among the three models. For example, in stage $\mathrm{F}$ as shown in Fig. 7, the fitted dielectric constant curves with CRIM and MWBH obtain slopes in low frequency range, which are obviously more gentle than that from NW model. Besides that, the upper limit of the dispersion below $100 \mathrm{MHz}$ fitted with CRIM and MWBH extend to higher frequencies, and the discrepancy from the experimental results of them are both larger than the NW model. Furthermore, the fitting effect of CRIM critically distorted in frequency range below $100 \mathrm{MHz}$, for it neglects the interaction between different components. Analogous phenomena could be found in the loss factor fitting and other experimental stages.

After the hydrate formation was completed in stage B, as shown in Fig. 6, only the dispersion of the THF hydrate can be observed. According to the 3D X-ray CT result in the NLL state, there was a slight amount of air (colored as black in Fig. 4(b)) between the solid blocks. This is because that the density decrement as the hydrate was forming was accompanied by a volume inflation and an exothermic process, and consequently, the gap between unconsolidated sand particles might possibly be extended. As the THF solution was consumed, air initially dissolved in the solution escaped from the liquid and filled the micro gaps between solid blocks[73, 74]. Therefore, in stage B and $\mathrm{D}$, the gas phase component has been added into the mixture, and except for stage A and F, there would be at least three kinds of phase components in the rest of the stages.

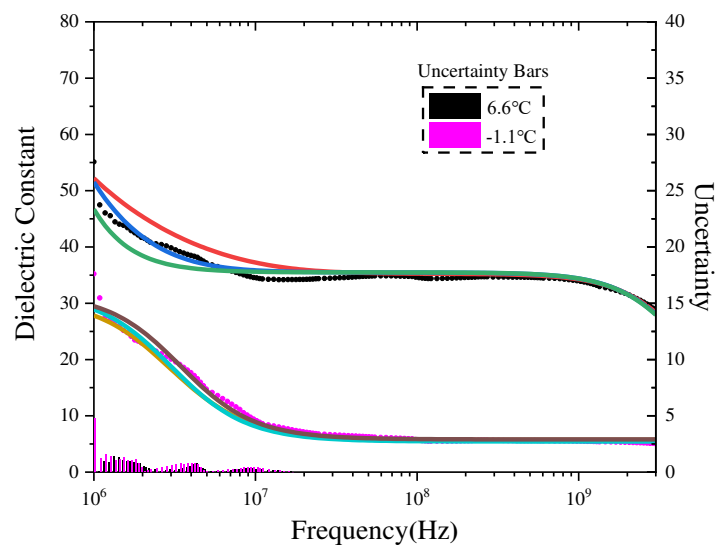

(a)

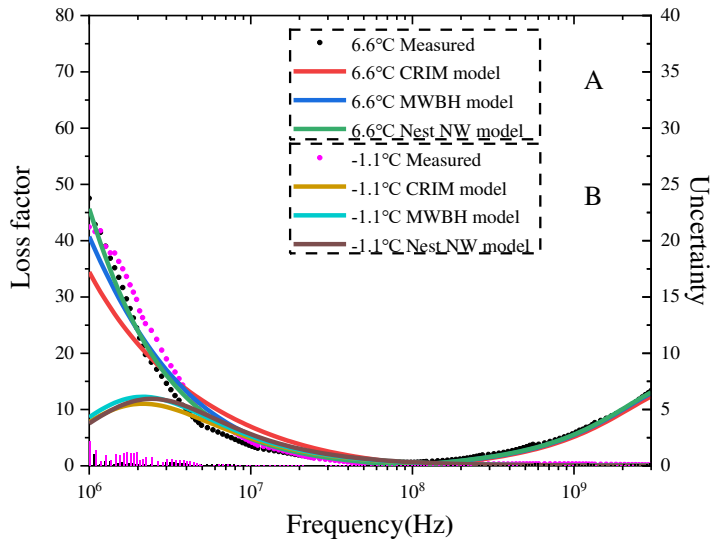

(b)

Fig. 6. The dot spectra are the complex relative permittivities in stage A and stage B. The uncertainties of the measured data are presented as bar graphs. The solid spectra are fitted to measure the permittivities with three models. The dielectric constant and loss factor are with a quartz sand particle size of about $0.9 \mathrm{~mm}$.

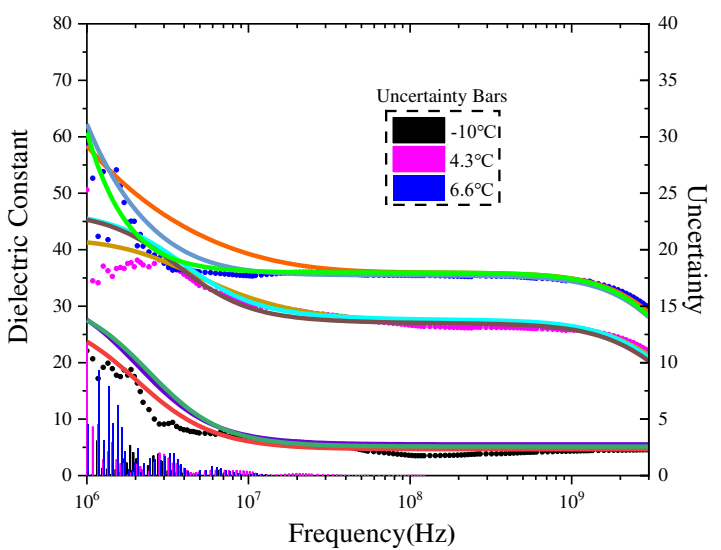

(a)

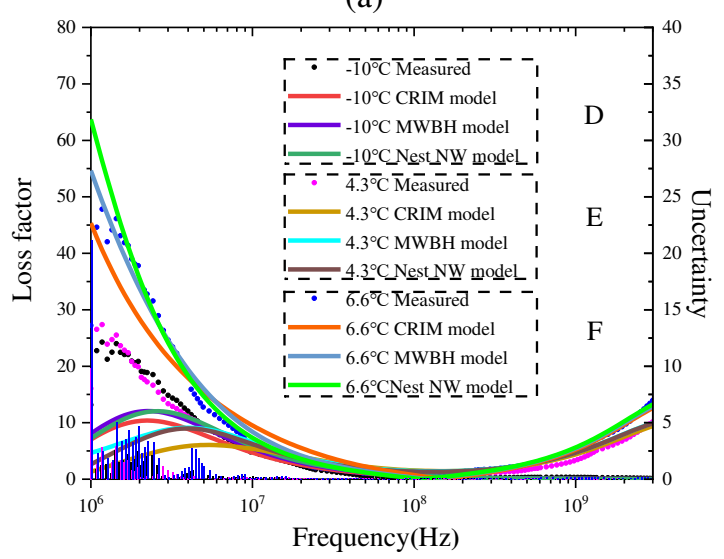

(b)

Fig. 7. The dot spectra are the complex relative permittivities in stage $\mathrm{D}$, stage $\mathrm{E}$, and stage $\mathrm{F}$. The uncertainties of the measured data are presented as bar graphs. The solid spectra are fitted to measure the permittivities with the three models. The dielectric constant and loss factor are with a quartz sand particle size of about $0.9 \mathrm{~mm}$.

Regarding the detailed parameters in Table III, both MWBH and the modified NW models described the dispersed phase (quartz sand in stage $\mathrm{A}$ and $\mathrm{F}$, hydrate coated quartz sand in stage $\mathrm{B}, \mathrm{D}$, and $\mathrm{E}$ ) as naked or confocal shelled spheres in different stages. $r$ and $d$ in millimeters are the radius of sand particle and hydrate shell thickness, respectively, and it should 
be noted that all the parameters were set as a spatially uniform value for the same condition. $\mathrm{r}$ was $0.45 \mathrm{~mm}$ all the time, and $\mathrm{d}$ always changed throughout the whole experiment. In stage B and $\mathrm{D}$, the hydrate shell was completely formed, and there was just a difference in temperature, so the shell thickness d remained the same. When it came to stage $\mathrm{E}$, the hydrate began to dissociate, at the specific time node $758^{\text {th }}$ min shown in Table III, the thickness d decreased to $0.092 \mathrm{~mm}$ and $0.105 \mathrm{~mm}$ in the MWBH and NW models, respectively. Moreover, liquid generated by the hydrate melting gradually accumulated, air bubbles were immediately filled with the fluid solution, and the gas phase vanished very early in stage E. The surface conductivity of the solid particle -- $\sigma_{\mathrm{PS}}$, was located at the outer shell of the solid spheres. Also, as the fraction of liquid solution grew, the double layer effect was enhanced, and the value of the surface conductivity increased. The surface conductivity $\sigma_{\mathrm{PS}}$ was introduced to the complex permittivity of the solid phase at the interface with a form of $-j \sigma_{P S} / \omega \varepsilon_{0}$. Specifically, in stage A and $\mathrm{F}$, the dielectric properties of quartz sand became, dependent on frequency, equal to $\varepsilon_{\text {snad }}-j \sigma_{P S} / \omega \varepsilon_{0}=4-j \sigma_{P S} / \omega \varepsilon_{0}$, and in stage $\mathrm{E}$, by making $\sigma$ in (17) equals to $\sigma_{P S}$, the complex permittivity of the hydrate, which acted as outer shells of the solid spheres, was redefined. In this section, the value of $\sigma_{P S}$ was obtained by fitting the optimum performance to the measured data.

Compared with the standard volumetric fractions obtained with X-ray CT, some advantages of the modified NW model were revealed. The deviations of the fitted result with the modified NW model was smaller than $1.5 \%$ compared to the standard values, and results calculated with the other two models were both much larger. For example, the volumetric fraction of quartz sand calculated with CRIM were always smaller than $40 \%$, which is obviously incorrect. Limited by the $\mathrm{X}$-ray equipment, we could not monitor the real-time X-ray images as the experiment was going on. Although only standard fractions at WHE and NLL states could be provided, we could still analyze the hydrate melting process in stage $\mathrm{E}$ by referring to the two states. It seems unusual that for the selected time node at stage $\mathrm{E}$, the estimated volumetric fraction of quartz sand was only about $1 / 3$ of the standard from X-ray CT. This is probably due to the flush effect of the melting solution which made the local microstructure different e.g. the fragile sand matrix collapsed and was reconstructed with bigger gaps containing more fluid. Eventually, the temporarily unstable fluid state resulted in the distinctions between stage $\mathrm{E}$ and other stages. For the three models, the mixture as homogeneous media, and the parameters in those models were obtained by normalizing the heterogeneous distribution in the microscopic view. In the beginning of the dissociation period, the solution liquid flushed over the surface of hydrate shells, where there was no surface charge. Also, as the hydrate melted, some shells collapsed and the surface of sand grains gradually contacted the solution, which had surface charges. Furthermore, as the liquid phase amount increased, the surface conduction apparently increased (see $\sigma_{\mathrm{PS}}$ from stage $\mathrm{E}$ to stage $\mathrm{F}$ ). As well as stage A, stage $\mathrm{F}$ was also in the WHE state, and the estimated volume ratios were nearly the same. For the same temperature condition, $\sigma_{P S}$ in stage $F$ was a little larger than in stage A, because the mobility of counterions located at the solid surface were slightly enhanced by the liquid flow, when the hydrate melted. As a result, we can draw the conclusion that, no matter at which experimental stage, the modified NW model obtained better accuracy than the CRIM and MWBH models, and the surface conduction modification helped to match the interfacial polarization below $100 \mathrm{MHz}$. Concerning both fitting effects and accuracy, the modified NW model showed its advantages, although as shown in Table III, the other two reference models possessed same $\sigma_{\mathrm{PS}}$ as applied to the proposed model. At the same time, the modified NW model can also provide physical mechanism explanations when compared with semi-empirical models [76].

TABLE III

PARAMETERS FITTED IN EACH STAGE

\begin{tabular}{|c|c|c|c|c|c|c|c|c|c|c|}
\hline Stage & "Temperature & $\begin{array}{l}\text { Time } \\
(\mathrm{min})\end{array}$ & Model & $\begin{array}{c}\text { Quartz } \\
\text { Sand }\end{array}$ & "Hydrate & Solution & Air & $\overline{\sigma_{\mathrm{PS}}(\mathrm{S} / \mathrm{m})}$ & $\begin{array}{ll}\mathrm{r}(\mathrm{mm}) \\
\end{array}$ & 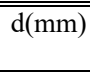 \\
\hline \multirow{3}{*}{ A } & \multirow{3}{*}{$6.6^{\circ} \mathrm{C}$} & \multirow{3}{*}{$44^{\text {th }}$} & CRIM & $38.0 \%$ & $0 \%$ & $62.0 \%$ & $0 \%$ & $4.2 \mathrm{e}-3$ & None & None \\
\hline & & & MWBH & $40.0 \%$ & $0 \%$ & $60.0 \%$ & $0 \%$ & $4.2 \mathrm{e}-3$ & 0.45 & None \\
\hline & & & NW & $43.7 \%$ & $0 \%$ & $56.3 \%$ & $0 \%$ & $4.2 \mathrm{e}-3$ & 0.45 & None \\
\hline \multirow{3}{*}{ B } & \multirow{3}{*}{$-1.1^{\circ} \mathrm{C}$} & \multirow{3}{*}{$228^{\text {th }}$} & CRIM & $39.8 \%$ & $60.1 \%$ & $0 \%$ & $0.1 \%$ & 0 & None & None \\
\hline & & & MWBH & $40.3 \%$ & $52.7 \%$ & $0 \%$ & $7.0 \%$ & 0 & 0.45 & 0.145 \\
\hline & & & NW & $43.0 \%$ & $55.0 \%$ & $0 \%$ & $2.0 \%$ & 0 & 0.45 & 0.147 \\
\hline \multirow{3}{*}{$\mathrm{D}$} & \multirow{3}{*}{$-10^{\circ} \mathrm{C}$} & \multirow{3}{*}{$320^{\text {th }}$} & CRIM & $39.9 \%$ & $60.1 \%$ & $0 \%$ & $0.1 \%$ & 0 & None & None \\
\hline & & & MWBH & $42.0 \%$ & $52.0 \%$ & $0 \%$ & $6.0 \%$ & 0 & 0.45 & 0.139 \\
\hline & & & NW & $43.0 \%$ & $55.0 \%$ & $0 \%$ & $2.0 \%$ & 0 & 0.45 & 0.147 \\
\hline \multirow{3}{*}{ E } & \multirow{3}{*}{$4.4^{\circ} \mathrm{C}$} & \multirow{3}{*}{$758^{\text {th }}$} & CRIM & $27.0 \%$ & $22.4 \%$ & $50.6 \%$ & $0 \%$ & $3.0 \mathrm{e}-5$ & None & None \\
\hline & & & MWBH & $29.4 \%$ & $22.0 \%$ & $48.6 \%$ & $0 \%$ & $3.0 \mathrm{e}-5$ & 0.45 & 0.092 \\
\hline & & & NW & $30.1 \%$ & $26.3 \%$ & $43.6 \%$ & $0 \%$ & $3.0 \mathrm{e}-5$ & 0.45 & 0.105 \\
\hline \multirow{3}{*}{$\mathrm{F}$} & \multirow{3}{*}{$6.6^{\circ} \mathrm{C}$} & \multirow{3}{*}{$896^{\text {th }}$} & CRIM & $37.1 \%$ & $0 \%$ & $62.9 \%$ & $0 \%$ & $6.5 \mathrm{e}-3$ & None & None \\
\hline & & & MWBH & $40.0 \%$ & $0 \%$ & $60.0 \%$ & $0 \%$ & $6.5 \mathrm{e}-3$ & 0.45 & None \\
\hline & & & NW & $43.5 \%$ & $0 \%$ & $56.5 \%$ & $0 \%$ & $6.5 \mathrm{e}-3$ & 0.45 & None \\
\hline
\end{tabular}

A typical example of the modified NW model's application on hydrate dissociation process is illustrated in Fig. 8. The forward direction of the timeline is from the left side to the right side, and regarding Fig. 5, the time axis could not be reversed.
At the beginning, the mixture in stage $\mathrm{D}$ is in the 'NLL' state, and the continuous background was air (white color in the picture), the dispersed inclusions were quartz sand balls (green color) with hydrate shells (yellow confocal shells). From the 
temperature curve depicted in Fig. 5, the hydrate dissociation was triggered by increasing temperature, and the melting process was time consuming in contrast to the momentary formation. So, from the middle segment of Fig. 8, an obviously gradual change appeared both in the background and the inclusion balls: pores were filled with the liquid solution (color from light blue to dark blue) by degrees, and the hydrate shell little by little became thinner. The temperature remained at $4.4^{\circ} \mathrm{C}$ all though the hydrate dissociation in stage $\mathrm{E}$, until there was no hydrate remaining. Then the mixture returned to the 'WHE' state as shown in the right segment of Fig. 8, and the yellow shells totally vanished. For the component configuration in unconsolidated porous media in this study, the modified NW model can provide an appropriate description of the phase transition process. Furthermore, concerning more complex natural sediments, as more components were added, the NW model with a single shell layer was extended to a multi-layer type as depicted in Fig. 1(b).

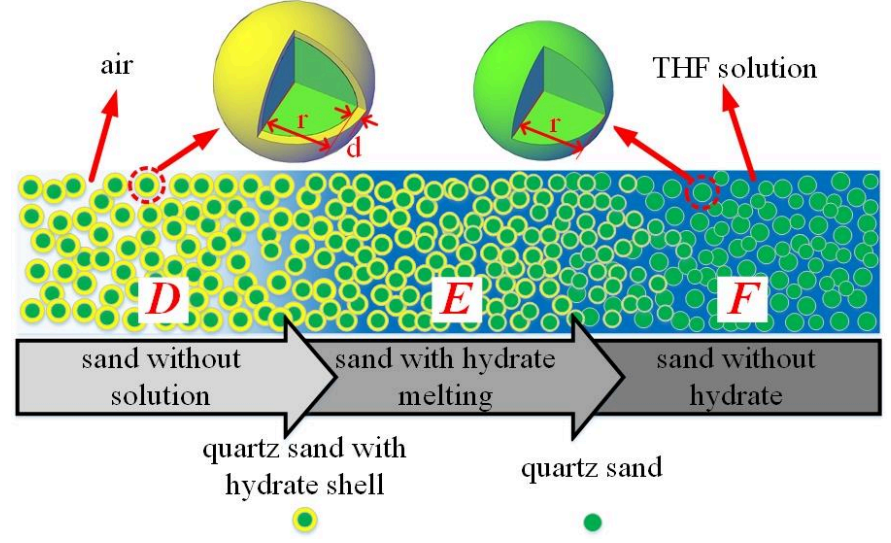

Fig. 8. Artist's interpretation schematic of how a hydrate dissociating process is fitted with the modified NW model in unconsolidated porous media. Green balls, yellow shells, white and blue background represent quartz sand particles, hydrate, air, and liquid solution respectively. The scale in the figure is just for purpose of model demonstration and does not represent the actual sizes.

\section{B. Model Application for Different Conditions}

Temperature: Considering that complex permittivity is always temperature dependent, and moreover as described in the previous section, there are close relationships between the mixture components and temperature for porous media containing hydrate. To illuminate the temperature influence on the proposed model, we used two situations as examples: temperature varying without phase transition and phase transiting at the invariable phase equilibrium temperature. For the first situation, the measured data and fitted results are illustrated in Fig. 9 at three different temperature points in stage B. As there was no liquid phase left, the dispersion peaks below $100 \mathrm{MHz}$ can only be from THF hydrate, and these peaks drifted to lower frequencies as the temperature fell. Viewing the fitted parameters exhibited in Table IV, volumetric parameters of every component remained invariant as the temperature fell. This is because that Cole-Cole parameters for THF hydrate are functions of temperature, and for static permittivity $\varepsilon_{0}$ (high frequency permittivity was constant $\varepsilon_{\infty}=7$ ) and relaxation time $\tau$, the relationship was shown as [38,65]:

$$
\varepsilon_{0}-\varepsilon_{\infty}=14900 / \mathrm{T}
$$

$\log (\tau)=-11.9+1265.7 / \mathrm{T}$

Applying the Cole-Cole parameters in the modified NW model according to varying temperatures, low frequency dispersion changed and the fitted curves well matched the experimental data, although there was no difference in the mixture's components and structure.

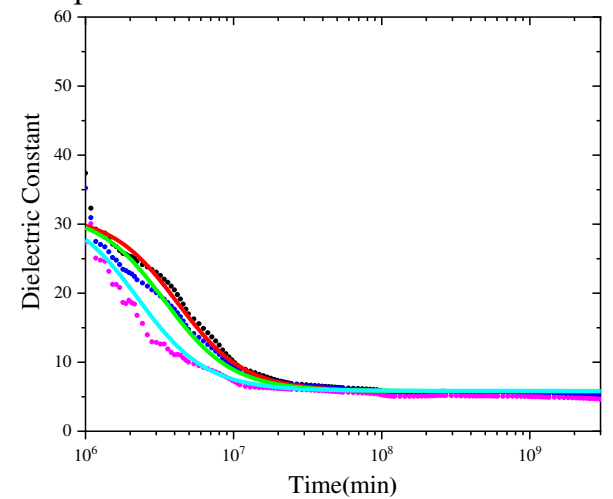

(a)

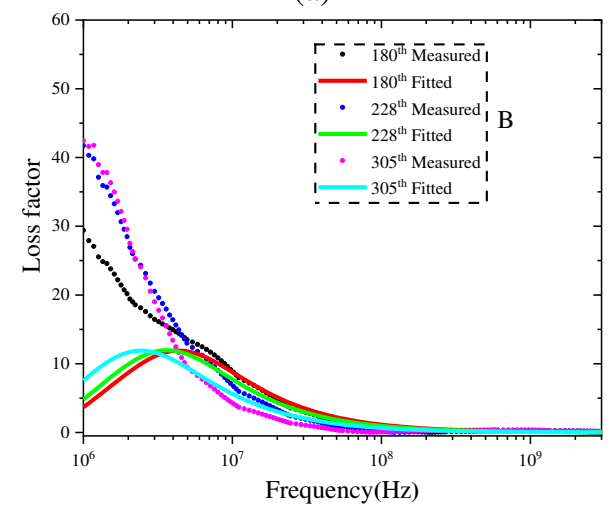

(b)

Fig. 9. The dot spectra are the measured complex permittivity at the same position in stage B. The solid spectra are fitted permittivity with the modified NW model.

For the second situation, the mixture in stage $\mathrm{E}$ was maintained at the equilibrium temperature of $4.4^{\circ} \mathrm{C}$ with the hydrate melting. Complex permittivity was selected at four time points in the dark grey area (between $746^{\text {th }}$ min and $758^{\text {th }}$ min, inside the probe's detection zone, the dissociation process mainly occurred in this time range) in Fig. 5, and the measured and fitted spectra are presented in Fig. 10. Both the dielectric constant and loss factor showed obvious differences at the four time points in the whole frequency range, while the variation tendencies were stable over time. The dielectric constant over $10 \mathrm{MHz}$ integrally decreased, but for the range below $10 \mathrm{MHz}$, the decrement became smaller as the frequency declined. The loss factor part over $100 \mathrm{MHz}$ grew, and the low frequency part shrank. The process of hydrate melting and liquid accumulation are clearly revealed in volume fractions changing in Table IV. Also, the slight increment of the quartz sand fraction can be perceived, and it showed a tendency approaching $43.5 \%$ (the sand volumetric percentage after dissociation was complete in stage E). To sum up, even if the temperature is stable, phase transition can be revealed from the parameters of the modified NW model, and combined with the temperature dependent 
Cole-Cole model, the modified NW model performed well in both situations with different temperature conditions.

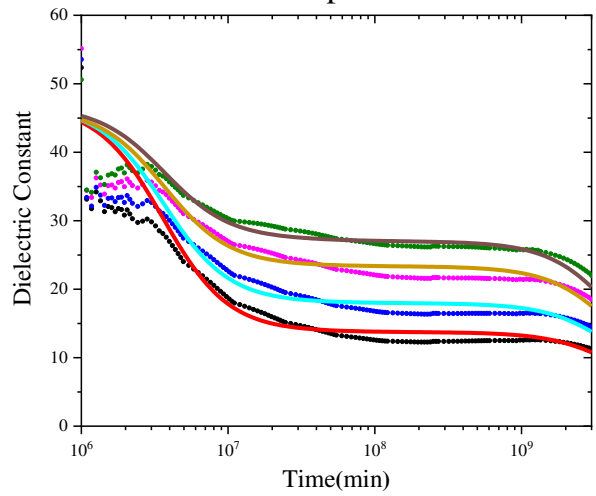

(a)

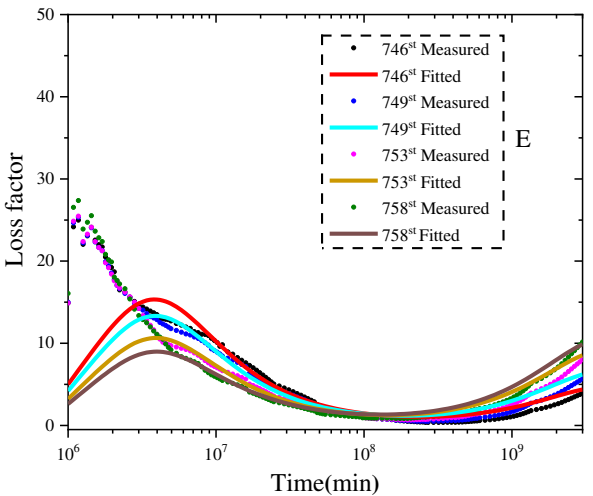

(b)

Fig. 10. The dot spectra are the measured complex permittivity at the same position in stage E. The solid spectra are fitted permittivity with the modified $\mathrm{NW}$ model. All the spectra were obtained at the equilibrium temperature of THF hydrate $4.4^{\circ} \mathrm{C}$.

TABLE IV

PARAMETERS FITTED IN STAGE B AND STAGE E

\begin{tabular}{cccccccc}
\hline \hline Stage & Temperature & Time $(\min )$ & Quartz Sand & Hydrate & Solution & Air & $\sigma_{\text {PS }}(\mathrm{S} / \mathrm{m})$ \\
\hline \multirow{3}{*}{ B } & $3^{\circ} \mathrm{C}$ & $180^{\text {th }}$ & $42.0 \%$ & $56.0 \%$ & None & $2.0 \%$ & None \\
& $-1.1^{\circ} \mathrm{C}$ & $228^{\text {th }}$ & $42.0 \%$ & $56.0 \%$ & None & $2.0 \%$ & None \\
& $-9^{\circ} \mathrm{C}$ & $305^{\text {th }}$ & $42.0 \%$ & $56.0 \%$ & None & $2.0 \%$ & None \\
\hline \multirow{4}{*}{ E } & $4.4^{\circ} \mathrm{C}$ & $746^{\text {th }}$ & $28.4 \%$ & $50.7 \%$ & $20.9 \%$ & None & $2.0 \mathrm{e}-6$ \\
& $4.4^{\circ} \mathrm{C}$ & $749^{\text {th }}$ & $28.8 \%$ & $46.3 \%$ & $28.9 \%$ & None & $5.0 \mathrm{e}-6$ \\
& $4.4^{\circ} \mathrm{C}$ & $753^{\text {th }}$ & $29.5 \%$ & $32.0 \%$ & $38.5 \%$ & None & $1.0 \mathrm{e}-5$ \\
& $4.4^{\circ} \mathrm{C}$ & $758^{\text {th }}$ & $30.1 \%$ & $26.3 \%$ & $43.6 \%$ & None & $3.0 \mathrm{e}-5$ \\
\hline \hline
\end{tabular}

Surface Conductance: There were interfaces between the liquid and solid in three stages A, E, and F. For stage A and F, interfaces occurred between the quartz sand and THF solution, while in stage $\mathrm{E}$, interfaces were between the outer surface of hydrate shells and the solution. Hence, as we illustrated in section II, we configured the modified NW model in that quartz sand in stage $\mathrm{A} / \mathrm{F}$ and hydrate in stage $\mathrm{E}$ possessed surface conductivity respectively. For the same temperature of $6.6^{\circ} \mathrm{C}$ at one measuring position, the differences between the complex spectra before hydrate formed and after hydrate completely melted can be found in Fig. 11. The fluid flush during the melting process increased the surface mobility, and as the fitted $\sigma_{P S}$ listed in Table III, the surface conduction in stage $\mathrm{F}\left(896^{\text {th }}\right.$ $\mathrm{min})$ was 1.5 times larger than in stage $\mathrm{A}\left(44^{\text {th }} \mathrm{min}\right)$ at $6.6^{\circ} \mathrm{C}$. Because the volumetric fractions were almost the same, spectra over $10 \mathrm{MHz}$ coincided in Fig. 11, while the larger surface conductivity in stage $\mathrm{F}$ caused a higher rise as the frequency decreased below $10 \mathrm{MHz}$. Then we considered the surface conduction changing with the hydrate melting in stage E. First, as the fitted results presented in Table III and IV show, the surface conductivity in stage $\mathrm{E}$ was smaller than in stages $\mathrm{A} / \mathrm{F}$ at the surface of the quartz sand, and the surface conductance occurred at the surface of hydrate shells in stage E. Second, although obvious growth was found in surface conduction with the hydrate dissociating, it can hardly act as counterweight to the decrease of the hydrate's characteristic dispersion. Referring to the fitted results with the modified NW model, a conclusion can be drawn that the surface conduction scarcely has an influence on bulk dielectric properties at low frequencies when the hydrate is in the process of dissociating with extremely low salinity, but nonetheless, when the mixture comes to the 'WHE' state, a different phenomenon emerges. Furthermore, it should be stated that, the conductivity of liquid in all experiments of this study are near the level of the deionized water, and under this prerequisite, surface conductance dominated in the M-W effect. Whether the effect of the liquid conductance surpass the surficial charges will be verified in our future works, if the liquid is mineralized.

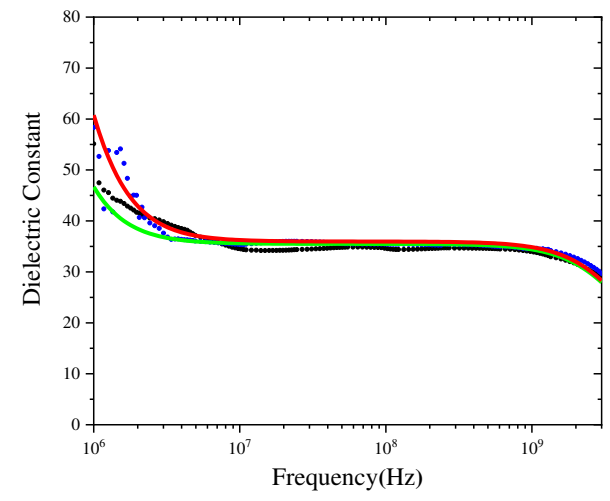

(a) 


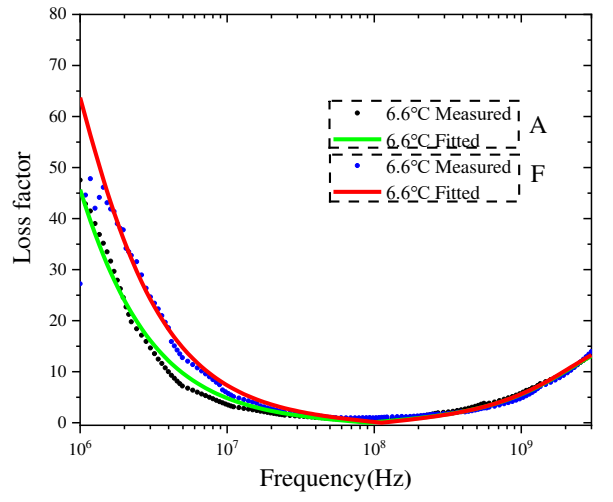

(b)

Fig. 11. Dot spectra are the measured complex permittivity at the same temperature $6.6^{\circ} \mathrm{Cy}$ in stage $\mathrm{A}$ and $\mathrm{F}$, respectively. The solid curves are fitted bulk permittivity with the modified NW model.

Particle Size: To investigate the influence of the porous media, another experiment of hydrate forming and dissociating inside the quartz sand with a different particle size with an average of $0.28 \mathrm{~mm}$ was carried out. Fig. 12 and 13 show the fitted curves using the modified NW model and corresponding measured permittivity in the 'NLL' and 'WHE' states. To control variables, the temperatures are the same in each figure. Considering the fluidity of the solution during the hydrate formation and dissociation processes, and to maximize the accuracy and spatial homogeneity of the measured data, permittivity in the 'WHE' state was measured in stage A, and the 'NLL' state was measured in stage C.

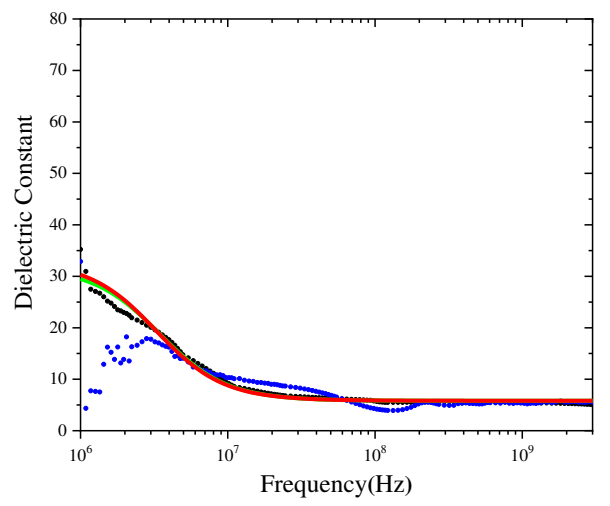

(a)

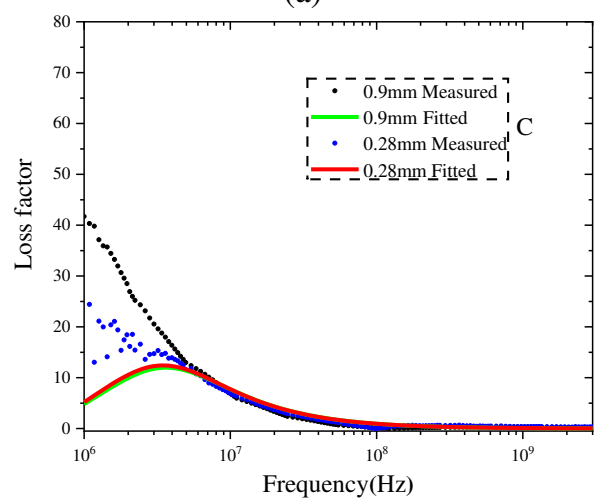

(b)

Fig. 12. The measured results and fitted performance according to two different particle sizes in the 'NLL' state. Dielectric constant and loss factor are presented in (a) and (b) respectively.
When the grain size of the sand is very uniform (sorting coefficient close to 1), porosity is almost grain size independent[77]. The fitting volumetric fractions in Fig. 12 and Fig. 13 are listed in Table IV, and there were no substantial differences according to the change of the particle size. Referring to the standard ratio in Table I, the error of the fitted results was $2.7 \%$. For the 'NLL' state, spectra of both the dielectric constant and loss factor of the two particle sizes significantly coincided in Fig. 12, and because there was no liquid phase, dispersion peaks below $100 \mathrm{MHz}$ were totally contributed by the formed THF hydrate, and the temperature and component ratio were almost the same.

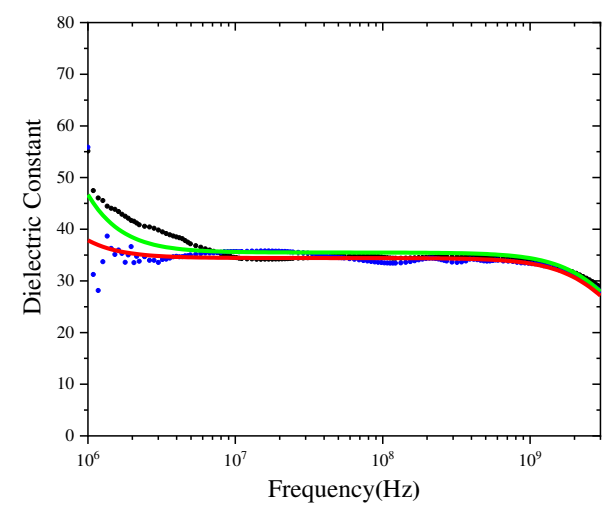

(a)

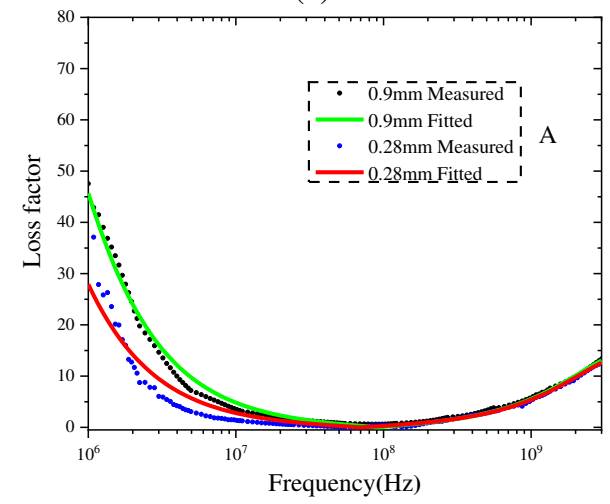

(b)

Fig. 13. The measured results and fitted performance according to two different particle sizes in the 'WHE' state. Dielectric constant and loss factor are presented in (a) and (b) respectively.

However, as shown in Fig. 13, the increment of the complex permittivity with particle size of $0.9 \mathrm{~mm}$ was larger as the frequency decreased below $100 \mathrm{MHz}$, when compared with particle size of $0.28 \mathrm{~mm}$. The overlapping parts in the frequency range over $100 \mathrm{MHz}$, and the very close fitted fraction ratios in Table IV indicated that the volumetric ratio was not the factor which made the permittivity vary, but the particle size was the critical cause. As previously analyzed on surface conductance, inherent parameters of the rock matrix but not the properties of the solution were the determinant for surface conductivity with low solution salinity[54]. Gaps between the quartz sands can be assumed as a micro capillary network, which can be uniformly quantified with geometrical parameters effective length $\boldsymbol{l}$, radius $\boldsymbol{r}_{\boldsymbol{c}}$, and effective cross section area $\boldsymbol{A}$. The surface conductivity $\sigma_{\mathrm{PS}}$ can be derived with the equation containing 
streaming potential $\Delta \boldsymbol{\Phi}$, streaming current $\boldsymbol{I}$, and solution electrical conductivity $\boldsymbol{\sigma}_{\text {solution }}[78,79]$ :

$$
\sigma_{P S}=\frac{2 \sum_{\mathrm{S}}}{r_{c}}=\frac{\boldsymbol{I} \cdot l}{A \triangle \Phi}-\sigma_{\text {solution }}
$$

The length of the capillary $\boldsymbol{l}$ becomes longer with a larger particle size. In the two experiments the conductivity of solution $\sigma_{\text {solution }}$ is negligible, and thus the surface conductivity $\sigma_{\mathrm{PS}}$ is in direct proportion to the capillary length $\boldsymbol{l}$. Concerning the surface conductivity of the 'WHE' state in Table IV, $\sigma_{\mathrm{PS}}$ with a particle size of $0.9 \mathrm{~mm}$ was about 1.8 times larger than that with a particle size of $0.28 \mathrm{~mm}$, which did not precisely match the size ratio of the two particles. This resulted from idealization of all the pore spaces as uniformed capillaries, however, in fact, they are not uniform. Not only that, the change of capillary's geometry caused by different particle sizes is not limited to length $\boldsymbol{l}$, and the section area $\boldsymbol{A}$ can also change. Although it is impossible and fruitless to evaluate the practical geometrical parameters of the pores, it is sure that the grain size can affect the complex permittivity of the mixture through the surface conductivity.

TABLE IV

PARAMETERS FITTED WITH DIFFERENT PARTICLE SIZE USING THE NW MODEL

\begin{tabular}{cccccccccc}
\hline \hline State & Temperature & $\begin{array}{c}\text { Particle } \\
\text { Size(mm) }\end{array}$ & $\begin{array}{c}\text { Quartz } \\
\text { Sand }\end{array}$ & Hydrate & Solution & Air & $\boldsymbol{\sigma}_{\mathrm{PS}}(\mathbf{S} / \mathbf{m})$ & $\mathbf{r}(\mathbf{m m})$ & $\mathbf{d}(\mathbf{m m})$ \\
\hline WHE & $6.6^{\circ} \mathrm{C}$ & 0.9 & $42.7 \%$ & $0 \%$ & $57.3 \%$ & $0 \%$ & $4.2 \mathrm{e}-3$ & None & None \\
\hline WHE & $6.6^{\circ} \mathrm{C}$ & 0.28 & $44.3 \%$ & $0 \%$ & $55.7 \%$ & $0 \%$ & $2.3 \mathrm{e}-3$ & None & None \\
\hline NLL & $-10^{\circ} \mathrm{C}$ & 0.9 & $42 \%$ & $56 \%$ & $0 \%$ & $2 \%$ & None & 0.45 & 0.147 \\
\hline NLL & $-100^{\circ} \mathrm{C}$ & 028 & $44 \%$ & $54.5 \%$ & $0 \%$ & $1.5 \%$ & None & 0.14 & 0.0431 \\
\hline
\end{tabular}

\section{CONCLUSION}

A physics based novel model for predicting the bulk complex permittivity of unconsolidated porous media containing hydrate was proposed and validated. The model was developed based on the nested Wagner's theory which was applied to dielectric properties estimation of a multi-component mixture, and it was modified by taking the solid surface conductance into account. Experimental results were obtained with deionized water, and thus the influence of salinity was diminished. In the proposed NW model, surface conductivity was assigned to the solid sphere, and the M-W polarization was believed to be enhanced by the surface conductance. In comparing with other existing models, a major improvement is that the proposed NW model has taken the physical mechanism during the hydrate formation and dissociation into consideration, while at the same time, improving the fitted performance with emphasis on the lowfrequency range (below 100MHz) where the hydrate dispersion and the interfacial polarization are located. Large discrepancies appeared in fitted results with the CRIM model, and it cannot appropriately match the experimental results in the frequency band below $100 \mathrm{MHz}$, although the fitted performance is acceptable in the higher frequency range. Referring to the standard values of volumetric fractions acquired with X-ray CT, the fraction estimation obtained with the modified NW model better agreed with experimental data than the MWBH model throughout the whole experimental process. Combined with the temperature dependent Cole-Cole parameters, the proposed model can relate the dielectric properties with the microscopic processes of component transformation at different temperature conditions. In addition, it was found that the dependence of the dielectric dispersion on surface conductance is significantly affected by the different experimental stages and particle sizes, especially in the low frequency range. Using the modified NW model established in this study, the hydrate saturation and volumetric fractions of other components can be successfully estimated from the measured complex permittivity spectra, and this model can provide a better fitted performance, compared with other common mixing methods. By adding more nested layers, this model can potentially be applied to unconsolidated media containing more components not only restricted to hydrate, but also including liquid and gas. It can be of practical significance for the exploration and exploitation of hydrate and other geophysical applications.

\section{REFERENCES}

[1] P. Englezos, "Clathrate hydrates," Industrial \& engineering chemistry research, vol. 32, no. 7, pp. 1251-1274, 1993.

[2] E. D. Sloan Jr and C. A. Koh, Clathrate hydrates of natural gases. CRC press, 2007.

[3] M. D. Max, Natural gas hydrate in oceanic and permafrost environments. Springer Science \& Business Media, 2003.

[4] Y. Zhu et al., "Gas hydrates in the Qilian mountain permafrost, Qinghai, Northwest China," Acta Geologica Sinica-English Edition, vol. 84, no. 1, pp. 1-10, 2010.

[5] M. B. Clennell, M. Hovland, J. S. Booth, P. Henry, and W. J. Winters, "Formation of natural gas hydrates in marine sediments: 1. Conceptual model of gas hydrate growth conditioned by host sediment properties," Journal of Geophysical Research: Solid Earth, vol. 104, no. B10, pp. 22985-23003, 1999.

[6] K. Yamamoto et al., "Operational overview of the first offshore production test of methane hydrates in the Eastern Nankai Trough," in Offshore Technology Conference, 2014: Offshore Technology Conference.

[7] T. S. Collett and J. Ladd, "19. Detection of gas hydrate with downhole logs and assessment of gas hydrate concentrations (saturations) and gas volumes on the Blake Ridge with electrically resistivity log data," Proceedings of the Ocean Drilling Program, Scientific Results, Texas A\&M University, College Station, TX, USA, vol. 164, 2000.

[8] M. Lee and T. Collett, "In-situ gas hydrate hydrate saturation estimated from various well logs at the Mount Elbert Gas Hydrate Stratigraphic Test Well, Alaska North Slope," Marine and Petroleum Geology, vol. 28, no. 2, pp. 439-449, 2011.

[9] J.-f. Li et al., "The first offshore natural gas hydrate production test in South China Sea," China Geology, vol. 1, no. 1, pp. 5-16, 2018.

[10] T. S. Collett et al., "Gulf of Mexico Gas Hydrate Joint Industry Project Leg II logging-while-drilling data acquisition and analysis," Marine and Petroleum Geology, vol. 34, no. 1, pp. 41-61, 2012.

[11] M. Lee and T. Collett, "Integrated analysis of well logs and seismic data to estimate gas hydrate concentrations at Keathley Canyon, Gulf of Mexico," Marine and Petroleum Geology, vol. 25, no. 9, pp. 924-931, 2008. 
[12] M. W. Lee and W. F. Waite, "Estimating pore-space gas hydrate saturations from well log acoustic data," Geochemistry, Geophysics, Geosystems, vol. 9, no. 7, 2008.

[13] Y. Sun and D. Goldberg, "Dielectric method of high-resolution gas hydrate estimation," Geophysical research letters, vol. 32, no. 4, 2005.

[14] Y. Sun, D. Goldberg, T. Collett, and R. Hunter, "High-resolution well-log derived dielectric properties of gas-hydrate-bearing sediments, Mount Elbert Gas Hydrate Stratigraphic Test Well, Alaska North Slope," Marine and Petroleum Geology, vol. 28, no. 2, pp. 450-459, 2011.

[15] U. Majumdar et al., "Semi-quantitative gas hydrate assessment from petroleum industry well logs in the northern Gulf of Mexico," Marine and Petroleum Geology, vol. 85, pp. 233-241, 2017.

[16] M. Pirrone et al., "A novel approach based on dielectric dispersion measurements to evaluate the quality of complex shaly-sand reservoirs," in SPE Annual Technical Conference and Exhibition, 2011: Society of Petroleum Engineers.

[17] N. V. Seleznev et al., "Applications of dielectric dispersion logging to oil shale reservoirs," in SPWLA 52nd Annual Logging Symposium, 2011: Society of Petrophysicists and Well-Log Analysts.

[18] J.-y. Lee, "Hydrate-bearing sediments: Formation and geophysical properties," Georgia Institute of Technology, 2007.

[19] J. Lee, T. S. Yun, J. Santamarina, and C. Ruppel, "Observations related to tetrahydrofuran and methane hydrates for laboratory studies of hydrate-bearing sediments," Geochemistry, Geophysics, Geosystems, vol. 8, no. 6, 2007.

[20] J. Lee, J. Santamarina, and C. Ruppel, "Mechanical and electromagnetic properties of northern Gulf of Mexico sediments with and without THF hydrates," Marine and Petroleum Geology, vol. 25, no. 9, pp. 884-895, 2008.

[21] J. Lee, J. C. Santamarina, and C. Ruppel, "Parametric study of the physical properties of hydrate-bearing sand, silt, and clay sediments: 1. Electromagnetic properties," Journal of Geophysical Research: Solid Earth, vol. 115, no. B11, pp. 1-9, 2010.

[22] S. Liu, T. Han, G. Hu, and Q. Bu, "Dielectric behaviors of marine sediments for reliable estimation of gas hydrate saturation based on numerical simulation," Journal of Natural Gas Science and Engineering, vol. 73, p. 103065, 2020.

[23] J.-H. Sa et al., "Hydrate management in deadlegs: Detection of hydrate deposition using permittivity probe," Energy \& Fuels, vol. 32, no. 2, pp. 1693-1702, 2018.

[24] K. Haukalid and K. Folgerø, "Broad-band permittivity measurements of formation of gas hydrate layers using open-ended coaxial probes," Energy \& Fuels, vol. 30, no. 9, pp. 7196-7205, 2016.

[25] K. Haukalid, K. Folgerø, T. Barth, and S. L. Fjermestad, "Hydrate formation in water-in-crude oil emulsions studied by broad-band permittivity measurements," Energy \& Fuels, vol. 31, no. 4, pp. 3793-3803, 2017.

[26] T. Jakobsen and K. Folgerø, "Dielectric measurements of gas hydrate formation in water-in-oil emulsions using open-ended coaxial probes," Measurement Science and Technology, vol. 8, no. 9, p. 1006, 1997.

[27] M. C. Dobson, F. T. Ulaby, M. T. Hallikainen, and M. A. El-Rayes, "Microwave dielectric behavior of wet soil-Part II: Dielectric mixing models," IEEE Transactions on Geoscience and Remote Sensing, no. 1, pp. 35-46, 1985.

[28] J. M. Wraith and D. Or, "Temperature effects on soil bulk dielectric permittivity measured by time domain reflectometry: Experimental evidence and hypothesis development," Water Resources Research, vol. 35, no. 2, pp. 361-369, 1999.

[29] K. W. Wagner, "Erklärung der dielektrischen nachwirkungsvorgänge auf grund maxwellscher vorstellungen," Archiv für Elektrotechnik, vol. 2, no. 9, pp. 371-387, 1914.

[30] S. S. Dukhin, V. N. Shilov, and J. Bikerman, "Dielectric phenomena and double layer in disperse systems and polyelectrolytes," Journal of the Electrochemical Society, vol. 121, no. 4, p. 154C, 1974.

[31] A. Revil and P. Glover, "Nature of surface electrical conductivity sandstones, and clays in natural sands," Geophysical Research Letters, vol. 25, no. 5, pp. 691-694, 1998.

[32] V. L. Mironov, L. G. Kosolapova, and S. V. Fomin, "Physically and mineralogically based spectroscopic dielectric model for moist soils," IEEE Transactions on Geoscience and Remote Sensing, vol. 47, no. 7, pp. 2059-2070, 2009.

[33] M. Davies and K. Williams, "Dielectric relaxation in clathrates," Transactions of the Faraday Society, vol. 64, pp. 529-548, 1968.

[34] Q. Zeng, J. Li, H. Huang, X. Wang, and M. Yang, "Polarization response of clathrate hydrates capsulated with guest molecules," The Journal of chemical physics, vol. 144, no. 20, p. 204308, 2016.

[35] Y. Chen and D. Or, "Geometrical factors and interfacial processes affecting complex dielectric permittivity of partially saturated porous media," Water resources research, vol. 42, no. 6, 2006.

[36] O. A. de Lima and M. M. Sharma, "A generalized Maxwell-Wagner theory for membrane polarization in shaly sands," Geophysics, vol. 57, no. 3, pp. 431-440, 1992.

[37] K. Haukalid and K. Folgerø, "Dielectric mixture models for hydrate formation and agglomeration," in 2018 12th International Conference on Electromagnetic Wave Interaction with Water and Moist Substances (ISEMA), 2018, pp. 1-9: IEEE.

[38] B. Wang, Z. Zhang, and L. Xing, "Study on the Formation and Disassociation Process of Hydrate in Porous Media by Permittivity Dispersion Measurement," IEEE Access, vol. 7, pp. 175362-175376, 2019.

[39] J. Santamarina, F. Francisca, T. Yun, J. Lee, A. Martin, and C. Ruppel, "Mechanical, thermal, and electrical properties of hydratebearing sediments," in AAPG Hedberg Conference: Gas hydrates: Energy resource potential and associated geological hazards. $A A P G$, Vancouver, BC, 2004.

[40] J. R. Birchak, C. G. Gardner, J. E. Hipp, and J. M. Victor, "High dielectric constant microwave probes for sensing soil moisture," Proceedings of the IEEE, vol. 62, no. 1, pp. 93-98, 1974.

[41] J. A. Huisman, S. S. Hubbard, J. D. Redman, and A. P. Annan, "Measuring soil water content with ground penetrating radar," Vadose zone journal, vol. 2, no. 4, pp. 476-491, 2003.

[42] W. E. Kenyon, "Texture effects on megahertz dielectric properties of calcite rock samples," Journal of Applied Physics, vol. 55, no. 8, pp. 3153-3159, 1984.

[43] J. C. Maxwell, A treatise on electricity and magnetism. Clarendon press, 1881 .

[44] T. Hanai, N. Koizumi, T. Sugano, and R. Gotoh, "Dielectric Properties of Emulsions.(II): Electrical Conductivities of $\mathrm{O} / \mathrm{W}$ Emulsions," 1961.

[45] T. Hanai, "Theory of the dielectric dispersion due to the interfacial polarization and its application to emulsions," Kolloid-Zeitschrift, vol. 171, no. 1, pp. 23-31, 1960.

[46] V. D. Bruggeman, "Berechnung verschiedener physikalischer Konstanten von heterogenen Substanzen. I. Dielektrizitätskonstanten und Leitfähigkeiten der Mischkörper aus isotropen Substanzen," Annalen der physik, vol. 416, no. 7, pp. 636664, 1935.

[47] C. Boned and J. Peyrelasse, "Etude de la permittivite complexe d'ellipsoides disperses dans un milieu continu. Analyses theorique et numerique," Colloid and Polymer Science, vol. 261, no. 7, pp. 600-612, 1983.

[48] K. Asami, "Characterization of heterogeneous systems by dielectric spectroscopy," Progress in polymer science, vol. 27, no. 8, pp. 16171659, 2002.

[49] H. Fricke, "The complex conductivity of a suspension of stratified particles of spherical or cylindrical form," The Journal of Physical Chemistry, vol. 59, no. 2, pp. 168-170, 1955.

[50] A. Irimajiri, T. Hanai, and A. Inouye, "A dielectric theory of "multistratified shell" model with its application to a lymphoma cell," Journal of theoretical biology, vol. 78, no. 2, pp. 251-269, 1979.

[51] L. Stepin, "Permittivity of a medium with inhomogeneous spherical inclusions," SOVIET PHYSICS TECHNICAL PHYSICS-USSR, vol. 9, no. 10 , pp. $1348-\&, 1965$.

[52] A. Revil and P. Glover, "Theory of ionic-surface electrical conduction in porous media," Physical review B, vol. 55, no. 3, p. $1757,1997$.

[53] A. Revil, "Effective conductivity and permittivity of unsaturated porous materials in the frequency range $1 \mathrm{mHz}-1 \mathrm{GHz}, "$ Water Resources Research, vol. 49, no. 1, pp. 306-327, 2013.

[54] A. Revil, "On charge accumulation in heterogeneous porous rocks under the influence of an external electric field," Geophysics, vol. 78, no. 4, pp. D271-D291, 2013.

[55] P. Leroy, A. Revil, A. Kemna, P. Cosenza, and A. Ghorbani, "Complex conductivity of water-saturated packs of glass beads," 
Journal of Colloid and Interface Science, vol. 321, no. 1, pp. 103117, 2008.

[56] A. Weller, L. Slater, and S. Nordsiek, "On the relationship between induced polarization and surface conductivity: Implications for petrophysical interpretation of electrical measurements," Geophysics, vol. 78, no. 5, pp. D315-D325, 2013.

[57] K. Titov, V. Komarov, V. Tarasov, and A. Levitski, "Theoretical and experimental study of time domain-induced polarization in water-saturated sands," Journal of Applied Geophysics, vol. 50, no. 4, pp. 417-433, 2002.

[58] C. Grosse, "Permittivity of a suspension of charged spherical particles in electrolyte solution. 2. Influence of the surface conductivity and asymmetry of the electrolyte on the low-and highfrequency relaxations," The Journal of Physical Chemistry, vol. 92, no. 13 , pp. 3905-3910, 1988.

[59] J. Schurr, "On the theory of the dielectric dispersion of spherical colloidal particles in electrolyte solution1," The Journal of Physical Chemistry, vol. 68, no. 9, pp. 2407-2413, 1964.

[60] A. Revil, K. Koch, and K. Holliger, "Is it the grain size or the characteristic pore size that controls the induced polarization relaxation time of clean sands and sandstones?," Water Resources Research, vol. 48, no. 5, 2012.

[61] A. Revil and M. Skold, "Salinity dependence of spectral induced polarization in sands and sandstones," Geophysical Journal International, vol. 187, no. 2, pp. 813-824, 2011.

[62] A. K. Jonscher, "The "universal'dielectric response," nature, vol. 267, no. 5613, pp. 673-679, 1977.

[63] F. E. Critchfield, J. A. Gibson Jr, and J. L. Hall, "Dielectric Constant and Refractive Index from 20 to $35^{\circ}$ and Density at $25^{\circ}$ for the System Tetrahydrofuran-Water1," Journal of the American Chemical Society, vol. 75, no. 23, pp. 6044-6045, 1953.

[64] B. Morris and D. Davidson, "A clathrate hydrate of cyclobutanone: dielectric relaxation of the host and guest molecules," Canadian Journal of Chemistry, vol. 49, no. 8, pp. 1243-1251, 1971.

[65] S. Gough, R. Hawkins, B. Morris, and D. Davidson, "Dielectric properties of some clathrate hydrates of structure II," The Journal of Physical Chemistry, vol. 77, no. 25, pp. 2969-2976, 1973.

[66] Y. P. Handa, "Effect of hydrostatic pressure and salinity on the stability of gas hydrates," Journal of Physical Chemistry, vol. 94, no. 6, pp. 2652-2657, 1990.

[67] K. K. Østergaard, R. Anderson, M. Llamedo, and B. Tohidi, "Hydrate phase equilibria in porous media: effect of pore size and salinity," Terra Nova, vol. 14, no. 5, pp. 307-312, 2002.

[68] Y. Mingjun, S. Yongchen, L. Yu, C. Yongjun, and L. Qingping, "Influence of pore size, salinity and gas composition upon the hydrate formation conditions," Chinese Journal of Chemical Engineering, vol. 18, no. 2, pp. 292-296, 2010.

[69] H. Martens, J. Reedijk, and H. Brom, "Measurement of the complex dielectric constant down to helium temperatures. I. Reflection method from $1 \mathrm{MHz}$ to $20 \mathrm{GHz}$ using an open ended coaxial line," Review of Scientific Instruments, vol. 71, no. 2, pp. 473-477, 2000.

[70] D. K. Misra, "A quasi-static analysis of open-ended coaxial lines (short paper)," IEEE Transactions on Microwave Theory and Techniques, vol. 35, no. 10, pp. 925-928, 1987.

[71] B. Filali, F. Boone, J. Rhazi, and G. Ballivy, "Design and calibration of a large open-ended coaxial probe for the measurement of the dielectric properties of concrete," IEEE Transactions on Microwave Theory and Techniques, vol. 56, no. 10, pp. 2322-2328, 2008.

[72] W. Ellison, "Permittivity of pure water, at standard atmospheric pressure, over the frequency range $0-25 \mathrm{THz}$ and the temperature range 0-100 C," Journal of physical and chemical reference data, vol. 36 , no. 1, pp. 1-18, 2007.

[73] S. Gough and D. Davidson, "Composition of tetrahydrofuran hydrate and the effect of pressure on the decomposition," Canadian Journal of Chemistry, vol. 49, no. 16, pp. 2691-2699, 1971.

[74] S. Takeya et al., "Imaging and density mapping of tetrahydrofuran clathrate hydrates by phase-contrast x-ray computed tomography," Applied physics letters, vol. 90, no. 8, p. 081920, 2007.

[75] C. Matzler, "Microwave permittivity of dry sand," IEEE Transactions on Geoscience and Remote Sensing, vol. 36, no. 1, pp. 317-319, 1998.

[76] R. L. Van Dam, B. Borchers, and J. M. Hendrickx, "Methods for prediction of soil dielectric properties: a review," in Detection and remediation technologies for mines and minelike targets $X, 2005$, vol. 5794, pp. 188-197: International Society for Optics and Photonics.

J. J. Rogers and W. B. Head, "Relationships between porosity, median size, and sorting coefficients of synthetic sands," Journal of Sedimentary Research, vol. 31, no. 3, pp. 467-470, 1961.

[78] J. Wang, H. Hu, W. Guan, and H. Li, "Electrokinetic experimental study on saturated rock samples: zeta potential and surface conductance," Geophysical Journal International, vol. 201, no. 2, pp. 869-877, 2015.

[79] J. Wang, "Experimental Study on Electrokinetic Effects in Porous Rock Samples and Measurements of P and S Wave Velocities While Drilling " Doctor of Engineering PhD Thesis, School of Astronautics, Harbin Institute of Technology, 2012.

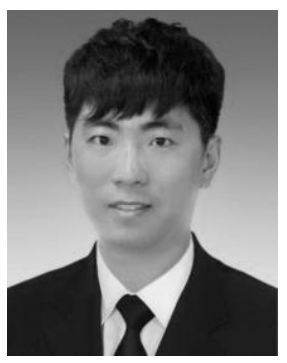

B. WANG (M'20) received the B.S. in applied physics from Qingdao University in 2010, and the Ph.D. degree in radio physics from Shandong University, China, in 2015 .

From 2015 to 2017, he was a lecturer with China University of Petroleum (East China). Since 2018, he has been an Associate Professor. His research interests include detection technology and automatic devices, permittivity measurement, the dielectric logging method, measurement of gas hydrate, and the numerical simulation method of electromagnetics.

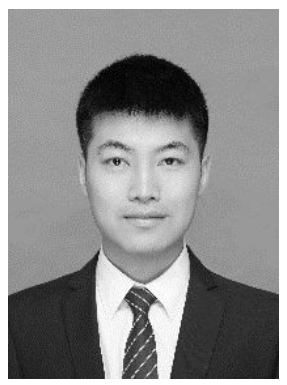

Z. ZHANG received the B.S. degree in building electrical and intelligent engineering in school of information and electrical engineering from Shandong Jianzhu University in 2017. He is currently pursuing the M.S. degree in college of control science and engineering at China University of Petroleum (East China), Qingdao, China. From 2017 to 2019, he was a postgraduate with China University of Petroleum (East China). His research direction was detection technology and automatic devices.

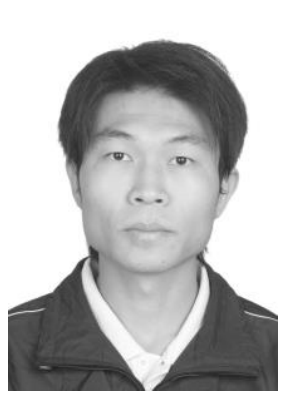

L. XING (M'17) received the B.S. in automation and M.S. degree in control science and engineering from China University of Petroleum (East China) in 2005 and 2008, respectively, and the $\mathrm{Ph} . \mathrm{D}$. degree in process system engineering from Cranfield University, $\mathrm{UK}$, in 2012.

From 2012 to 2015, he was a lecturer with China University of Petroleum (East China). Since 2016, he has been an Associate Professor. His research interests include detection technology and automatic devices, computer measurement and control systems, 
measurement of gas hydrate and multiphase flow, and multiphysical field coupling numerical simulation methods.

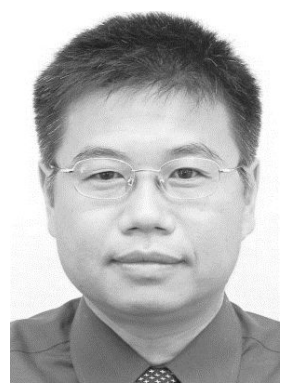

L. Lao received the B.Sc. and M.Sc. degrees in the measurement science and technology, and Ph.D. degree in the process and control engineering from ZhejiangUniversity, China, in 1983, 1986, and1998, respectively. In 1986, he joined the Department of Electrical Measurement and Instrumentation, China Institute of Metrology where he became a Lecturer in 1988 and an Associate Professor in 1997. After obtaining his Ph.D., he joined the Institute of Mechanics, Chinese Academy of Sciences as a postdoctoral researcher studying gas/liquid two-phase flows in horizontal pipes. Since 2007, he has been with Cranfield University, where he was a Research Fellow in the School of Engineering, and became a Senior Research Fellow in 2012. Currently he is a Senior Research Fellow in the Centre for Thermal Energy Systems and Materials. Prior to joining Cranfield University, he worked in the area of two phase flows in closed channels in Imperial College London as a Research Associate for four years and a Research Fellow in the University of Cambridge for two years. He is a Member of Institute of Physics. Dr Lao's current research interests cover process measurement \& systems, and experimental fluid dynamics of multiphase flow systems. His expertise embraces both sensing technologies and multiphase fluid dynamics. 